\title{
Subcoalition Cluster Analysis: A New Method for Modeling Conflict in Organizations
}

\author{
Scott C. Ganz \\ Georgetown McDonough School of Business and AEI \\ scott.ganz@georgetown.edu \\ Daniel Schiff \\ Georgia Tech School of Public Policy \\ schiff@gatech.edu
}

\begin{abstract}
The theory that firms are coalitions of competing interests is a cornerstone of multiple streams of research in management theory, many of which are as old as the field itself. However, there are relatively few quantitative studies that examine the dynamics of coalition building, conflict, and compromise inside of organizations. One important reason for this gap between theory development and empirical study is a lack of quantitative methods for identifying the fault lines that define intraorganizational conflict. In our paper, we propose Subcoalition Cluster Analysis (sCCA) as one such method. sCCA uses data from a set of agents who report preferences over pairs of alternatives to reveal a partition of agents that defines meaningful, representative, and stable subcoalitions. The partition of agents corresponds to an equilibrium in which each subcoalition's preferences reflect those of their members and each member is assigned to the subcoalition whose preferences they best align with. We then analyze three cases in which an organization or community faced the possibility of coalition-based conflict in order to show how sCCA can be used to study the social structure of conflict systems in a variety of empirical settings.
\end{abstract}

Key words: Organizational studies : Decision making; Organizational studies : Behavior; Organizational studies : Goals; Programming : Linear : Applications

\section{Introduction}

The theory that firms are coalitions of competing interests is a cornerstone of multiple streams of research in management theory, many of which are as old as the field itself (Cyert and March 1963. Pfeffer and Salancik 2003, March and Olsen 1989, Zald and Berger 1978, Mintzberg 1983). Originating in the Carnegie School and the Behavioral Theory of the Firm (BTOF), coalition-based theories propose that collective decisions emerge from a bottom-up process of intrafirm conflict and compromise among interest groups - coined subcoalitions in Cyert and March (1963) — rather 
than from a common interest in profit maximization or the fiat of a dictatorial executive (March 1962). Over the past 60 years, the organizational coalition concept has remained a pillar of mesoorganizational theories in subfields as diverse as Resource Dependence Theory (Pfeffer and Salancik 1974), Neoinstitutional Theory (Fligstein 1993), and Upper Echelon Theory (Hambrick and Mason 1984).

Despite the ubiquity of the coalition concept, there are relatively few quantitative studies that examine the dynamics of coalition building, conflict, and compromise inside organizations (Gavetti et al. 2007, Mithani and O'Brien 2020). One important reason for this gap between theory development and empirical study is a lack of quantitative methods for identifying the fault lines that define intraorganizational conflict. Quantitative researchers seeking to uncover the latent structure of conflict in organizations face interrelated computational and conceptual issues. First, many coalitional games in which self-interested agents organize themselves into distinct groups are known to lack stable, equilibrium outcomes (Ray and Vohra 2015). For those coalitional games for which stable groups do exist, the explosive number of possible coalitions when the number of agents grows beyond a handful often makes them computationally infeasible to discover (Chalkiadakis et al. 2011, Aziz et al. 2016). Although there are only around 50 ways to partition five agents, there are over 100 thousand ways to partition 10 agents and over 50 billion ways to partition 20 agents.

Second, the organizational coalition concept utilized in many management subfields has traveled a great distance from the original BTOF vision of organizations as characterized by distinct groups of agents with internally-consistent, externally-conflicting preferences over organization-level outcomes. In their recent review of the literature, Mithani and O'Brien (2020) identify eight behavioral attributes of coalition-based conflict in other management subfields that depart from the BTOF definition, including whether subcoalitions are defined by formal structure or roles (instead of preferences), whether they are are externally-oriented (instead of internally-oriented), and whether agents attach to subcoalitions in order to attain individual goals, such as power, influence, or economic rents (instead of attaching based on an agent's alignment with the subcoalition's preferences) (see also Mintzberg 1983, Stevenson et al. 1985).

The recent 50-year anniversary of the Carnegie School and the related resurgence of interest in neo-Carnegie ideas has renewed enthusiasm that these "divergent conceptualizations of coalitions" across the various management subfields actually "represent contextual manifestations of a common concept" (Mithani and O'Brien 2020, p. 3). A necessary condition for finding common conceptual ground, however, is the development of quantitative methods that permit researchers to evaluate these competing theories in data. We propose Subcoalition Cluster Analysis (sCCA) as one such method. sCCA uses data from a set of agents with preferences over pairs of alternatives. It reveals a partition of agents into subcoalitions. Each subcoalition has a "consistent preference 
ordering defined over the possible states of the system" (March 1962, p. 663), which we refer to as a collective weak order over alternatives. Each subcoalition's collective weak order is determined by a social welfare function that derives collective preferences from the individual preferences of its members. And, each agent's subcoalition membership is determined by the alignment between their individual preferences and each subcoalition's preferences. Furthermore, we introduce a series of computational methods that can be applied to sCCA in order to determine the number of subcoalitions that is most consistent with the data. In addition to helping analysts identify a partition of the recommended size, sCCA also reveals information about each subcoalition's preferences, membership, and relative size, the strength of intrasubcoalition consensus and intersubcoalition conflict, and the strength of the attachment of each agent to their assigned subcoalition.

What differentiates sCCA from other methods is that it satisfies three necessary requirements for theorizing an organization as a conflict system as defined in March (1962). Specifically, subcoalitions have preferences that are meaningful, representative, and stable. The first requirement means that subcoalitions have preferences that can be represented by a weak order over alternatives. The second is that the subcoalition's preferences are derived from the preferences of their membership. The third requires that subcoalitions and their preferences can be treated as fixed (and identified independently from the higher-level outcomes of conflict among subcoalitions). While these requirements may appear intuitive, they restrict the set of permissible quantitative methods considerably. In particular, the subcoalitions returned by a partition satisfying all three requirements must assign agents to the subcoalition whose preferences best align with the agent's while simultaneously defining the preferences of subcoalitions in terms of the outcome of this assignment process. This requires the identification of an equilibrium that is consistent with (1) the manner in which subcoalitions' preferences are defined given their membership, (2) given these subcoalition preferences, the manner in which agents' preferences over subcoalitions are determined, and (3) given each agent's preferences over subcoalitions, laws-of-motion that govern how agents move between them.

Existing community detection and clustering algorithms that define partitions of agents based upon pairwise correlations of agents' preferences fail to satisfy these criteria. Because these methods do not define a preference ordering over alternatives for each cluster, the revealed clusters are neither meaningful nor representative. Further, attempts to impose a social welfare function for the cluster or utility function for agents ex post often reveal that the proposed conflict system is not stable, because agents prefer to defect to subcoalitions to which they are not currently assigned.

Building sCCA on the BTOF vision of the organization-as-conflict-system has benefits and costs. One benefit is that, because alternative theories of coalition-based decision making tend to use the BTOF view as a jumping-off point, many of the core BTOF assumptions are also shared by 
these other theories (Mithani and O'Brien 2020). A second benefit arises from the mathematical and computational tractability of the BTOF's vision of coalition formation. Specifically, March (1962) proposes that "as a reasonable approximation" the structure of subcoalitions are "causally antecedent, and independent of, the decisions in the larger system" (p. 664-5). As described in detail in the paper, it is this assumption that makes it possible for sCCA to overcome many of the problems of equilibrium non-existence and computational complexity identified in prior research on coalitional games.

These assumptions also restrict sCCA's power to address a number of questions of interest to management researchers. sCCA's bottom-up vision of subcoalition formation makes it a better fit for settings in which decisions emerge organically from populations of agents with diverse organization-level goals than settings in which agents strategically engage in internal politicking for their own individual benefit. sCCA thus brackets strategic considerations related to power and the allocation of scarce resources that give rise to internal conflict (Pfeffer and Salancik 2003). sCCA also ignores the influence of roles, rules, hierarchy, and social institutions on subcoalition formation, except to the extent that they influence individual agent preferences (Cohen 1984, Hambrick and Mason 1984, March and Olsen 1989, Fligstein 1990). Further, individual preferences are assumed to be independent of the process of mobilizing agents into subcoalitions and also independent of conflict among subcoalitions over organization-level outcomes (Mintzberg 1983). For these reasons, we suspect that some management researchers would take issue with the claim that sCCA identifies subcoalitions at all and would instead argue that sCCA returns "latent coalitions[:] combinations of organizational participants with common interests who may interact around work-related issues and provide a potential pool of coalition members" (Stevenson et al. 1985, p. 264).

Nevertheless, we believe that those management researchers who might prefer to append an $l$ for "latent" to sCCA should still find sCCA to be a useful tool for quantitative research. For researchers seeking to test theories of coalition-based decision making that expand upon the BTOF model by adding strategic action, hierarchical relationships, formal roles, social institutions, and mobilization processes, the BTOF-based prediction returned by sCCA remains a useful baseline model. That is, in order to identify the effect of these other social and economic forces, it is necessary to begin with a theory of conflict systems in which agent preferences are exogenous and agents are grouped solely based on agreement about desired organizational outcomes. Akin to the way that the median voter theorem in political science provides a baseline prediction about collective choices under unidimensional preferences and majority voting (Black 1948) that legislative scholars have utilized to study the impact of rules (Krehbiel 1997), agenda-setting (Baumgartner and Jones 2009), and political parties (Cox and McCubbins 1993) on legislative outcomes, sCCA offers a clear prediction 
about subcoalition structure conditional on agent preferences that can be used to test the validity of richer theories about politics and power in organizations.

The paper proceeds as follows. We begin by placing sCCA in the context of existing theories and methods used to describe conflict in organizations. Then, we introduce the axiomatic foundations of sCCA, focusing in particular on how subcoalitions determine their collective preferences, how agents evaluate subcoalitions, and the characteristics of stable partitions of agents. Next, we describe the sCCA algorithm. In this section, we demonstrate how the partition of agents is discovered for a fixed number of subcoalitions and also offer several methods for determining the number of subcoalitions that is most consistent with the data.

We then analyze three cases in order to demonstrate sCCA's usefulness to management research in a variety of empirical settings. Each case represents a problem faced by an organization or community characterized by bottom-up conflict in which agents have diverse beliefs, preferences, or goals. The first case replicates an effort by a popular organizational sociology blog to analyze a crowdsourced community ranking of sociology departments, and evaluates the organizer's fear that a small group of unrepresentative respondents was biasing the ranking. The second analyzes the emergence of intraorganizational conflict at Wikipedia over how best to update the editing platform, and shows how the failure to identify the schism between experienced and inexperienced editors led the platform to underestimate the resistance to a strategic change intended to make the platform more inviting for novice editors. The third examines the emergence of conflict among baseball writers over whether candidates suspected of using performance-enhancing drugs deserve induction into the National Baseball Hall of Fame and also explores how social influence processes made this rift self-reinforcing.

\section{Relationship to Existing Research and Methods}

sCCA brings together the theory of coalition-based decision making from March (1962), a formal framework for defining stable partitions of agents into subcoalitions from coalitional game theory, and a computational approach for identifying these partitions from unsupervised machine learning.

The social structure of the conflict system revealed by sCCA is defined in March (1962) as "characterized by...elementary units that...can be described as having a consistent preference ordering," and where "the most preferred states of all elementary units cannot be simultaneously realized" (p. 663). We build on this definition so that it is also consistent with the concept of a hedonic coalition structure, which is a theory of group formation in which "the payoff to a player depends only on the composition of members of the coalition to which she belongs" Bogomolnaia and Jackson 2002, p. 202). In our case, the agent's payoff is a function of the alignment of the agent's preferences with their assigned subcoalition's collective weak order (which is itself defined by the 
individual preferences of its members). This reimagining of March (1962) permits the structure of the conflict system to be defined as an equilibrium to a hedonic game, i.e., a stable partition among goal-oriented agents who desire to attach to the groups that make them best off.

Defining the process of subcoalition formation in this manner permits the sCCA algorithm to build on prior research on coalitional game theory in economics (Ray and Vohra 2015) and computational social choice Aziz et al. 2016). The literature on hedonic games, for example, identifies a variety of equilibrium concepts that depend on assumptions about the rationality of individual agents and the types of defections from the existing coalition structure that are permitted. However, equilibrium existence is only guaranteed for a small subset of hedonic gamesand, even then, only for equilibria robust to unilateral deviations (Bogomolnaia and Jackson 2002, Ray and Vohra 2015). Further, for many hedonic games in which equilibria are known to exist, efficient algorithms for discovering them are not (Aziz et al. 2016). Lastly, there is often no way of systematically determining whether an equilibrium for a hedonic game exists other than by exhaustively searching over all possible partitions. For many non-hedonic coalitional games, e.g., games in which the payoffs to agents depend on the competitive strategies of other groups, these problems of equilibrium non-existence and computational complexity are further exacerbated (Ray) and Vohra 2015).

This difficulty of uncovering stable partitions of agents, much less knowing whether stable partitions exist, reinforces the need to ground sCCA in a theory of coalition-based conflict that is known to have an equilibrium solution that can be reliably discovered. Standard game-theoretic models fail on both counts. Axelrod et al. (1995) make this point in their empirical study of standard-setting alliances. "For complex alliance composition problems," the authors argue, "it is virtually impossible to determine complete payoff functions as game theory traditionally requires" (p. 1497). As we describe in subsection 3.1, an important feature of the March (1962) conception of subcoalition-based conflict is that there always exists a stable partition of agents into subcoalitions and search algorithms to quickly find them, given certain assumptions about the structure of the hedonic game and for particular equilibrium concepts.

However, the simplicity of the BTOF conception of coalition-based decision making brings with it important trade-offs. First, sCCA defines subcoalitions independently from the manner in which the conflict among the subcoalitions is eventually resolved. Unlike coalition-based conflict in Resource Dependence Theory, which predicts that the fault lines of conflict are determined by the desire for power inside the organization, or in Neoinstitutional Theory, which focuses on how externallydefined social institutions impact decision-making routines inside the firm, subcoalition structures defined by sCCA are independent of considerations related to power, legitimacy, rivalry, or hierarchy (Mintzberg 1983). Also, there are no side payments or other inducements for agents who remain 
in a subcoalition or are part of a winning subcoalition, as is common in game-theoretic treatments of coalition formation in which agents have transferrable utility (see, e.g., Von Neumann and Morgenstern 1944, Roth 1988). Finally, the BTOF model of coalition formation implies a decidedly bottom-up theory of organizational goal-setting: there is no executive broker (Thompson 1967) or top management team (Hambrick and Mason 1984) determining what constitutes a desirable outcome for a firm.

This bottom-up description of the social institutions that shape conflict is shared by other recent methodological innovations in computational social science - and, in particular, cultural sociologythat also partition agents based upon their preferences. Most similar to sCCA are Relational Class Analysis (RCA) (Goldberg 2011) and Correlational Class Analysis (CCA) (Boutyline 2017), two methods that identify the latent dimensions along which cultural conflict emerge, referred to as cultural schemas. Whereas sCCA bases common group membership on agreement about desired outcomes, RCA and CCA base common membership on agreement about the nature of conflict over outcomes. The following example from Boutyline (2017) illustrates the difference: Imagine two types of agents. One type likes pop, blues, and rock, strongly likes classical and opera, and is indifferent toward bluegrass and country. The other is indifferent toward pop, blues, and rock, strongly dislikes classical and opera, and strongly likes bluegrass and country. sCCA would identify these two groups as maximally dissimilar, because their musical tastes are diametrically opposed. RCA and CCA would identify the groups as maximally similar, because they share schemas about the nature of the disagreement over musical tastes. Now, imagine a third type of agent, which prefers pop, blues, and rock to classical and opera, and dislikes bluegrass and country. If forced to divide the agents into two groups, sCCA would align the first with the third. RCA and CCA, in contrast, would align the first with the second.

We view sCCA and RCA/CCA as complementary methods. Depending on the research question, the analyst may desire to capture latent disagreement over the desirability of alternatives directly or latent disagreement about the dimensionality of inter-group conflict. In a study of legislative decision making, for example, sCCA would reveal competing party platforms whereas RCA/CCA would reveal the policy issues that define the left-right axis. Further, any dataset capable of being analyzed by RCA or CCA can straightforwardly be analyzed by sCCA as well.

Researchers familiar with RCA/CCA or similar community detection methods (e.g., Newman 2006) may wonder whether a similar community-detection approach could be designed for the task of identifying subcoalitions if negatively correlated observations were treated as being maximally different instead of maximally similar. On the one hand, this algorithm would successfully place agents in communities with others with correlated preferences over alternatives. On the other, these communities would not be subcoalitions, because the identified communities of agents would be 
unable to be characterized as having subcoalition-level preferences. Some social welfare function that aggregates the community preferences into a collective weak order could be subsequently applied, but upon aggregating the community's preferences there is no reason to believe that agents would be assigned to the communities that make them best off. In other words, the process of making the subcoalition meaningful and representative is likely to also make the subcoalition unstable.

Additionally, although sCCA can analyze any data suitable for RCA/CCA, the converse is not true. Community detection methods that rely on pairwise distances between numerical arrays that represent agents' preferences also require that agents' preferences are complete. In observational settings where the number of alternatives under consideration is large, algorithms like these are often impractical. An important advantage of sCCA is that it returns stable subcoalitions given sparse data on agent preferences without first imputing preferences or imposing priors upon agents with missing data (see, e.g., Salganik and Levy 2015). How sCCA handles missing data is described in greater detail in the discussion of Informative Social Choice in subsection 3.2 and in the first case analysis in subsection 4.1 .

\section{Analyzing intraorganizational Conflict using sCCA}

Next, we describe sCCA in detail. First, we analyze how the partition of agents returned by the sCCA algorithm reflects the concept of conflict systems defined in March (1962), focusing in particular on the axiomatic properties of stable partitions. Then, we describe the computational approach implemented in sCCA for discovering the subcoalitions, as well as a series of methods for revealing the number of subcoalitions consistent with the data.

\subsection{Axiomatic Foundations}

sCCA is an algorithm that returns the solution to a hedonic game in which all agents seek to maximize their utility when choosing among subcoalitions and in which subcoalitions are defined by a partition of agents into distinct subsets (Bogomolnaia and Jackson 2002). The payoff for each agent is determined by their relative affinity for their subcoalition's collective weak order over alternatives. These conceptions of subcoalitions and agent preferences offer an intuitively appealing definition of subcoalition stability, which we call weak stability: a partition is weakly stable if there are no subcoalition members with preferences that are better aligned with another subcoalition's preferences than they are with their own subcoalition's preferences.

One way to ensure that a weakly stable subcoalition exists is to require that the process through which subcoalitions determine their respective collective weak orders-i.e., the social welfare function - is consistent with the manner in which individual agents evaluate the desirability of each subcoalition's collective weak order. A class of social welfare functions that have this consistency 
property are simple ranking scoring functions, or SRSFs (Conitzer et al. 2009). A social welfare function is an SRSF if the collective weak order returned by the social welfare function corresponds to the solution to an aggregate utility maximization problem, where each agent's utility is increasing in the extent to which the agent's preferences and the collective weak order are aligned.

What makes this class of social welfare functions so attractive for the problem of identifying subcoalitions is that, for any number of subcoalitions, there is guaranteed to be a partition of the agents which maximizes aggregate utility. Further, by examining the what-if utility for each agent if they were in a different subcoalition, we can efficiently test whether a candidate partition is stable and, if not, move an agent to a subcoalition with a more preferable collective weak order in order to discover a partition with higher aggregate utility.

This and other indented sections are intended for readers who are interested in more precise mathematical representations of concepts described in the body of the paper. Other readers may skip ahead.

Define a vote $v_{i, a, a^{\prime}}$ as encoding a preference relation for agent $i \in N$ between alternative $a$ and alternative $a^{\prime}$, where $N$ is a finite set of agents. If $v_{i, a, a^{\prime}}=1$, then agent $i$ weakly prefers $a$ to $a^{\prime}$. In contrast, if $v_{i, a, a^{\prime}}=0$, agent $i$ does not weakly prefer $a$ to $a^{\prime}$. If $v_{i, a, a^{\prime}}=1$ and $v_{i, a^{\prime}, a}=0$, then, $i$ strictly prefers $a$ to $a^{\prime}$. If $v_{i, a, a^{\prime}}=0$ and $v_{i, a^{\prime}, a}=1, i$ strictly prefers $a^{\prime}$ to $a$. If $v_{i, a, a^{\prime}}=1$ and $v_{i, a^{\prime}, a}=1, i$ is indifferent between the two alternatives. Finally, if $v_{i, a, a^{\prime}}=0$ and $v_{i, a^{\prime}, a}=0$ then $i$ expresses no preference over the two alternatives.

Define $V_{i}$ as the multiset union of all votes recorded for agent $i$ and $\mathbf{V}$ as the sum over all $V_{i}$. A multiset union records both the elements of the set and the instances of each element, called multiplicities. The sum of two multiset unions is a multiset union in which multiplicities are added for common elements.

Define a social welfare function as a function $f$ that maps from $\mathbf{V}$ to a collective weak order over the set of alternatives $f(\mathbf{V}) \in R(A)$, where $R(A)$ is the the set of all possible collective weak orders over alternatives $A$. A social welfare function $f$ is an SRSF if there exists a utility function $u$ such that for all $\mathbf{V}, f(\mathbf{V})=\arg \max _{r \in R(A)} \sum_{i \in N} \sum_{u_{i, a, a^{\prime}} \in V_{i}} u\left(v_{i, a, a^{\prime}}, r\right)$.

\subsection{Informative Social Choice}

Many familiar social welfare functions are also SRSF's. However, all of the social welfare functions in frequent use face drawbacks that make them a poor fit for identifying stable partitions of agents in observational data. Foremost, many SRSFs require that agents submit strict orders over all alternatives: ties or missing data are not permitted. In many observational settings, data satisfying these conditions may not exist. Further, especially when the set of alternatives grows large, it is impractical to require that participants make fine-grained distinctions over all pairs of 
alternatives. Even methods that permit agents to report weak orders implicitly require that agents have transitive preferences, an assumption that the analyst may not wish to impose upon the data. For instance, all positional scoring functions, including plurality rule, anti-plurality rule, and the Borda count require agents to have transitive preferences (Conitzer et al. 2009).

A second problem with many SRSF's is computational in nature. The one widely-studied SRSF that does not require that voters submit complete weak orders, called the Kemeny rule, is based on the following utility function: an agent receives one unit of utility for every pairwise evaluation of alternatives that is consistent with the pairwise ranking implied by the collective weak order. Unfortunately, the Kemeny rule is NP-hard to compute (Bartholdi et al. 1989). That is, finding the Kemeny ranking that maximizes aggregate utility requires evaluating an exponentially-growing set of potential collective weak orders over alternatives. With just 10 alternatives, there are over 100 million potential weak orders. With 15, there are more than 200 trillion. When combined with the number of potential partitions of agents, the combinatorics get massive, rendering the Kemeny rule impractical in many empirical contexts.

As a result, sCCA uses a recently proposed social welfare function called the $I_{2}$ rule Atkinson and Ganz 2021). The $I_{2}$ rule is an informative social welfare function and also an SRSF. The logic behind informative social welfare functions is that they seek to reveal the best-fitting collective weak order to the data after discarding uninformative noise, similar to linear regression in inferential statistics. Two additional features of the $I_{2}$ rule that are useful for sCCA are (1) it reveals collective weak orders in the presence of sparse data and (2) it is able to be computed very quickly using standard algorithms for solving integer linear programs.

The utility function associated with the $I_{2}$ rule is defined as follows: If an agent prefers alternative $a_{1}$ to $a_{2}$ and the collective weak order also prefers $a_{1}$ to $a_{2}$, then this pairwise comparison contributes zero to the agent's utility. If an agent prefers $a_{1}$ to $a_{2}$ and the collective weak order does not prefer $a_{1}$ to $a_{2}$, then the agent's utility is penalized in proportion to the extent of the violation. If an agent is indifferent between $a_{1}$ and $a_{2}$, it is treated as if the agent prefers $a_{1}$ to $a_{2}$ and prefers $a_{2}$ to $a_{1}$. Specifically, if we characterize the collective weak order as assigning alternatives to integer ranks, where higher ranked alternatives are preferred to lower ranked alternatives and where equallyranked alternatives indicate indifference, then an agent who prefers alternative $a_{1}$ to alternative $a_{2}$ contributes $-\max \left(0, \rho\left(a_{2}\right)-\rho\left(a_{1}\right)+1\right)$ to aggregate utility, where $\rho\left(a_{1}\right)$ and $\rho\left(a_{2}\right)$ represent the integer ranks of alternative $a_{1}$ and $a_{2}$, respectively, This utility function is also known as the agony function (see, e.g., Gupte et al. (2011) and Tatti (2017)). 
A social welfare function $f$ is an informative social welfare function if there exists a partition of the votes $\mathbf{V}=\mathbf{X}+\mathbf{E}$ and a corresponding collective weak order $f(\mathbf{V})$ with the following four properties:

1. There is no signal in the noise: The set of votes in $\mathbf{E}$ constitutes a neutral configuration, defined as a set of votes for which the aggregate number of votes in favor of an alternative is equal to the aggregate number of votes opposed to an alternative (summed over all pairwise comparisons and for all alternatives).

2. There is no noise in the signal: The cardinality of the set of votes in $\mathbf{E}$ is maximized.

3. The collective weak order respects the signal: For any two alternatives $a, a^{\prime} \in A$, if the number of votes for $a$ exceeds the number of votes for $a^{\prime}$ in any partition of votes $\mathbf{X}$ that satisfies the first two properties, then $a$ is strictly preferred to $a^{\prime}$ in the collective weak order $f(\mathbf{V})$.

4. The collective weak order accepts ties: Changing any set of strict preference relations to indifference relations in $f(\mathbf{V})$ would violate $(3)$, ceteris paribus.

For some sets of votes $\mathbf{V}$, there exist multiple collective weak orders that satisfy the above properties. The $I_{2}$ rule therefore adds a fifth property to ensure that the weak order returned is unique:

5. If all voters submit weak orders over alternatives, then $f(\mathbf{V})$ is the weak order consistent with the prior four properties for which all alternatives lose to as many other alternatives as possible. We use the $I_{2}$ rule - as opposed to the $I_{1}$ rule, which corresponds to the collective weak order for which all alternatives beat as many other alternatives as possible-because in settings with missing data the $I_{2}$ rule "pushes down" the rank of alternatives for which few pairwise comparisons are observed. In other settings, e.g., those in which the analyst prefers to have many highly-ranked alternatives or in which agents report complete preferences over alternatives, the $I_{1}$ rank would be more appropriate. Except in conditions with sparse voting data, however, both return similar collective weak orders and, as a result, subcoalitions.

As is shown in work by one of the authors that draws on prior research on combinatorial optimization (see, e.g., Ford and Fulkerson 1962, Ahuja et al. 1993, Williamson 2019), the utility function that the $I_{2}$ rank maximizes takes the following form: First, redefine a collective weak order $r \in R(A)$ in terms of a set of integer ranks $\rho \in \mathbb{Z}^{+}$such that $\rho(a, r)>\rho\left(a^{\prime}, r\right)$ if $a$ beats $a^{\prime}$ in $r$ and $\rho(a, r)=\rho\left(a^{\prime}, r\right)$ if $a$ ties $a^{\prime}$ in $r$. Then, $u\left(v_{i, a, a^{\prime}}, r\right)=-\max \left(0, \rho\left(a^{\prime}, r\right)-\right.$ $\rho(a, r)+1)$. Accordingly, define the utility for agent $i$ given collective weak order $r$ to be $u\left(V_{i}, r\right)=\sum_{v_{i, a, a^{\prime}} \in V_{i}} u\left(v_{i, a, a^{\prime}}, r\right)$. 


\subsection{Defining Stable Partitions}

Next, we explore characteristics of subcoalition stability. We begin with the concept of weak stability, a property shared by all partitions returned by sCCA. We also introduce two other stability concepts - Nash stability and Pareto stability - and compare each to weak stability in order to motivate a number of decisions about the design of the sCCA algorithm.

We define a partition as weakly stable if each agent prefers the collective weak order associated with their assigned subcoalition at least as much as they prefer the collective weak orders of the subcoalitions to which they are not assigned. Weak stability has desirable behavioral, mathematical, and computational properties. Behaviorally, by assuming that agents determine their fit with each subcoalition by evaluating its collective weak order rather than the subcoalition's membership itself, weak stability decreases the information necessary for agents to navigate the subcoalition structure in a goal-oriented way. If agents were instead required to evaluate their fit with the members of subcoalitions directly, they would need to know the preferences and subcoalition assignments of every agent. Mathematically, weak stability is desirable because a weakly stable partition exists for any set of agents, alternatives, preferences, and number of subcoalitions when the $I_{2}$ rule is used to determine collective weak orders, and when agents are assigned to subcoalitions that maximize their individual utility. Computationally, weak stability is desirable because any iterative process of actors "defecting" to subcoalitions whose collective weak orders they prefer followed by these new subcoalitions "renegotiating" their collective weak order according to the $I_{2}$ rule will always converge to a weakly stable partition.

Let $\pi$ be a partition of all agents $N$ that assigns each agent to one of $K$ subcoalitions $S_{k}(\pi) \subseteq$ $N$, where $k \in\{1, \ldots, K\}$ indexes subcoalitions. Define $\mathbf{V}_{k}$ as the aggregated votes for all agents in $S_{k}$ for partition $\pi$. Thus, $\mathbf{V}_{k}(\pi)=\sum_{i \in S_{k}(\pi)} V_{i}$. Let $\Pi$ represent all of the potential partitions of $N$ and $\Pi^{*}$ be the set of partitions that satisfy weak stability. Then, $\pi \in \Pi^{*} \Longleftrightarrow$ $u\left(V_{i}, f\left(\mathbf{V}_{k}(\pi)\right) \geq u\left(V_{i}, f\left(\mathbf{V}_{k^{\prime}}(\pi)\right), \forall k: i \in S_{k}(\pi)\right.\right.$ and $\forall k^{\prime}: i \notin S_{k^{\prime}}(\pi)$. Finally, define a weakly stable partition $\pi^{*}$ as any member of $\Pi^{*}$.

To see that a weakly stable partition always exists, note that the partition that maximizes aggregate utility for a given $K$, denoted $\pi_{K}^{* *}$, is weakly stable. If $\pi_{K}^{* *}$ were not weakly stable, then an agent could defect to a different subcoalition and be strictly better off without changing their new subcoalition's collective weak order, which contradicts the claim that the prior partition maximizes overall utility in the first place.

To see that a defection-and-renegotiation algorithm always finds a weakly stable partition, note that aggregate utility is strictly decreasing in every defection stage and weakly decreasing in every renegotiation stage. Thus the algorithm will either conclude upon discovering $\pi_{K}^{* *}$ or at a different weakly stable $\pi^{*}$ along the way. 
However, the weak stability concept has some drawbacks, as well. In particular, by implicitly assuming that actors cannot predict how the collective weak orders would change upon renegotiation if they defect, the weak stability concept permits certain knife-edge coalition structures and low-quality aggregate outcomes. For example, imagine there are four agents $A, B, C$, and $D$ and two alternatives $a_{1}$ and $a_{2}$. Agents $A$ and $B$ prefer $a_{1}$ to $a_{2}$. Agents $C$ and $D$ prefer $a_{2}$ to $a_{1}$. A partition that places $A$ and $C$ in one subcoalition and $B$ and $D$ in the other subcoalition would be weakly stable, as both would have collective weak orders that express indifference between $a_{1}$ and $a_{2}$. As a result, none of the four agents could do strictly better off with the collective weak order of the opposing subcoalition. This is a low-quality, knife-edge solution, because if any of the four agents were to defect from their assigned subcoalition, they would become the pivotal voter in their new subcoalition and the renegotiated collective weak order would perfectly align with their preferences. Further, if the defector were forward-looking, they would also see that they make all of the agents better off by defecting, because the preferences of the subcoalition that the defector exited would now be completely aligned with those of the "loser" in their new subcoalition. This would lead the loser in the new subcoalition to themselves defect, producing a final utility-maximizing partition in which $A$ and $B$ are in one subcoalition and $C$ and $D$ are in the other.

Alternative stability concepts overcome these disadvantages of weak stability, but at a cost in terms of behavioral validity and computational tractability. Nevertheless, we introduce the concepts of Nash stability and Pareto stability in order to help inform the interpretation of sCCA's results and because they highlight design features of the sCCA algorithm that lead knife-edge and lowquality partitions to be unlikely to be returned.

Like weak stability, Nash stability requires that partitions be robust to defection by any individual agent. However, unlike weak stability, Nash stability assumes that agents can predict the post-defection renegotiated collective weak orders of the subcoalitions they join. This foresight means that knife-edge cases characterized by collective weak orders with many ties are unlikely to satisfy Nash stability. Note that any Nash stable coalition is also weakly stable by definition, since if an agent can be made better off by defecting absent renegotiation, then they will also be better off defecting after renegotiation. Further, in order for a weakly stable coalition to not be Nash stable, the agent must be pivotal over some pair of alternatives, otherwise the collective weak order would be unchanged post-defection. In practice, this means that if the organization is indeed characterized by well-defined clusters of preferences, weakly stable coalitions will also tend to be Nash stable.

However, Nash stability has a number of behavioral and computational limitations. Behaviorally, it requires that agents know the preferences of all agents in all subcoalitions in order for them to predict post-renegotiation collective weak orders. Also, there is neither a guarantee for the agent 
that their unilateral defection to a new subcoalition would itself be Nash stable, nor a guarantee that a Nash stable partition that emerged after iterations of defection and renegotiation would be better for the agent than the original partition was. Computationally, Nash stable partitions may not exist and, if they do, we do not have efficient computational methods for discovering them. However, we can confirm that a given partition is Nash stable reasonably quickly by checking if defection to any other subcoalition leads to a better outcome agent-by-agent. For this reason, we recommend (when feasible) that researchers also evaluate whether the partition returned by sCCA is Nash stable. If so, it provides another level of confidence that the partition is behaviorally valid. In addition, thoughtful initialization of the sCCA algorithm that places agents with conflicting preferences into different subcoalitions helps avoid knife-edge weakly stable partitions and increases the likelihood that the subcoalitions returned by sCCA are Nash stable.

Another related stability concept is Pareto stability. A partition is Pareto stable if there does not exist any other partition producing the same number of subcoalitions such that at least one agent is made strictly better off and no agents are made worse off. The limitations of Pareto stability are different from those of Nash stability. On the one hand, there does exist a Pareto stable partition given our setup, because the partition that maximizes aggregate utility is also Pareto optimal by construction. 1 On the other, there is no efficient algorithm for finding a Pareto stable partition or way to check if a given partition is Pareto stable unless all potential partitions are enumerated.

The fact that the partition with the highest aggregate utility is Pareto stable, nevertheless, highlights the desirability of finding a partition for which aggregate utility is as close to its global maximum as possible. For sufficiently small sets of agents, this can be achieved by exhaustively searching over all of the possible partitions producing a specified number of subcoalitions. However, the number of potential partitions quickly exceeds computational limits for even modestly-sized numbers of agents. For larger sets of agents, we instead recommend replicating the algorithm many times with different initial subcoalitions and then selecting the partition with the highest aggregate utility in order to return a good approximation of a Pareto stable partition.

\subsection{The sCCA algorithm}

We next describe the sCCA algorithm. In brief, the algorithm is a function that takes in the set of all agents' binary preferences over alternatives and a pre-specified number of subcoalitions $K$, and returns a weakly stable partition of agents $\pi_{K}^{*}$. The algorithm is also designed to identify partitions that are not knife-edge (and are thus likely to be Nash stable) and that have high aggregate utility (meaning they are also likely to be a good approximation of a Pareto stable partition). After presenting the algorithm, we describe a set of methods drawn from prior research on unsupervised machine learning that can help analysts determine the best-fitting value of $K$ given the data.

\footnotetext{
${ }^{1}$ To see this, note that if aggregate utility is maximized and one agent is made strictly better off by a different
} partition, then a different agent must be made strictly worse off. 
3.4.1. Constructing binary preference data One of the advantages of sCCA is its permissive data requirements. All that is needed is that the data can be transformed into a series of binary relations among alternatives for which an agent can prefer alternative $a_{1}$ to alternative $a_{2}$, prefer $a_{2}$ to $a_{1}$, be indifferent between $a_{1}$ and $a_{2}$, or make no comparison between $a_{1}$ and $a_{2}$. Thus any data that can be transformed into an adjacency matrix over alternatives, where a 1 in a cell $\left(a_{1}, a_{2}\right)$ represents a weak preference for $a_{1}$ over $a_{2}$, can be analyzed using sCCA.

For example, in a survey in which respondents are asked to compare alternatives $a_{1}$ and $a_{2}$, when a respondent chooses option $a_{1}$ instead of $a_{2}$, the adjacency matrix for that respondent records a 1 in the $\left(a_{1}, a_{2}\right)$ cell and a 0 in a $\left(a_{2}, a_{1}\right)$ cell. If the respondent chooses $a_{2}$ instead of $a_{1}$, this pattern is reversed. If the respondent expresses indifference between the alternatives, a 1 is recorded in both the $\left(a_{1}, a_{2}\right)$ cell and $\left(a_{2}, a_{1}\right)$ cell. And, in settings where respondents only compare selected pairs of alternatives or in which they may decline to make a comparison, missing data is represented by a 0 in both the $\left(a_{1}, a_{2}\right)$ and $\left(a_{2}, a_{1}\right)$ cells.

More structured sources of preference data are also easily managed. If agents submit a ranked list of alternatives, this can straightforwardly be transformed into an adjacency matrix recording a 1 in all cells $\left(a_{1}, a_{2}\right)$ when $a_{1}$ is better ranked than $a_{2}$. A consideration for the analyst, in these cases, is whether two alternatives ranked on the same level indicate indifference (in which case both $\left(a_{1}, a_{2}\right)$ and $\left(a_{2}, a_{1}\right)$ record $\left.1 \mathrm{~s}\right)$ or no comparison (in which case both $\left(a_{1}, a_{2}\right)$ and $\left(a_{2}, a_{1}\right)$ record $\left.0 \mathrm{~s}\right)$. In practice, this decision only matters in cases with extremely sparse data. Along the same lines, if the respondent allocates points among or scores various alternatives, such as through use of a Likert scale, these data can be similarly transformed into a matrix in which alternatives allocated more points are preferred to those with fewer points (and the same judgment about alternatives allocated the same number of points applies). A setup where a respondent reveals their relative utility among alternatives, for example by indicating their willingness-to-pay, can be represented as an adjacency matrix in the same fashion.

3.4.2. sCCA's structure sCCA is structured as a set of nested loops, including a body, inner loop, and outer loop.

The body of the algorithm initializes a partition defining $K$ subcoalitions and then uses an iterative process of defection and renegotiation to search for a weakly stable partition. The body of the algorithm is a variant of Lloyd's algorithm, which is commonly referred to as the $k$-means algorithm (Hastie et al. 2009). For this reason, when discussing the body of sCCA, we adopt the convention of referring to the defection step as the assignment step and the renegotiation step as the updating step. The body of the algorithm concludes when a weakly stable partition associated with $K$ subcoalitions is discovered, which we denote $\pi_{K}^{*}$. 
The inner loop replicates the body of the algorithm $B$ times, each time with a different initialized partition of $K$ subcoalitions. Following the completion of the inner loop, then, the body has discovered $B$ weakly stable partitions. The inner loop returns the partition with the highest aggregate utility out of this set, denoted $\hat{\pi}_{K}^{*}$. The outer loop then evaluates the inner loop for varying values of $K$, where the set of possible $K$ is denoted $\mathcal{K}$. When the outer loop concludes, the algorithm has returned one $\hat{\pi}_{K}^{*}$ for each value of $K$. Finally, as discussed in more detail in subsubsection 3.4.8, in cases where the analyst is interested in identifying the number of subcoalitions most consistent with the data, $K^{*}$, the analyst may apply an additional set of routines in order to estimate $\hat{\pi}_{K^{*}}^{*}$. We refer to this as the $K^{*}$ function.

Figure 1 is a visual depiction of the flow of the algorithm. The nested boxes depict the nested loops of the algorithm, with the top of each box indicating how many iterations of the inner boxes are executed as part of the loop and the bottom of the box representing the object that is returned once the loop is complete.

Figure 1 Flow of the sCCA algorithm

$K^{*}$ function: This function determines the $K$ most consistent with the data, denoted $K^{*}$.

Outer Loop:

For each $K \in \mathcal{K}$, where $\mathcal{K}$ is the set of potential $K$ :

Inner Loop:

For each $b$ in $1, \ldots, B$, where $B$ is the the number of replications:

Body: The body searches for a weakly stable partition, denoted $\pi_{K}^{*}$

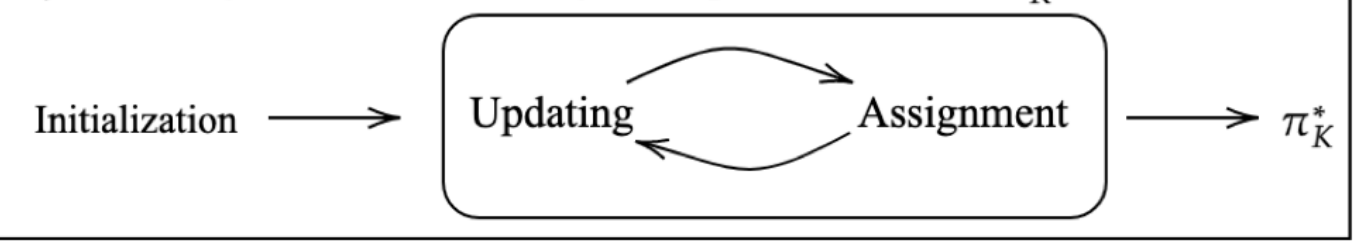

Return: $\widehat{\pi}_{K}^{*}$, the weakly stable partition with the highest aggregate utility.

Return: $\widehat{\pi}_{K}^{*}$ for all $K \in \mathcal{K}$.

Return: $\widehat{\pi}_{K^{*}}^{*}$, the highest-utility, weakly stable partition associated with $K^{*}$.

Next, we describe each of the parts of the sCCA algorithm in greater detail, focusing on the implementation of each of the algorithm's steps. 
3.4.3. Body: Subcoalition initialization The body of the algorithm begins by initializing a partition of agents. Although the algorithm will discover a weakly stable coalition for any initial partition, it is preferable to begin the updating-and-assignment process with an intelligent partition, i.e., one that avoids knife-edge solutions and reduces the number of updating-and-assignment steps required until a weakly stable partition is discovered.

We use a variant of the the "k-means++" seeding algorithm, which has been shown to achieve both of these goals when Lloyd's algorithm is used in geometric clustering contexts. Specifically, the computational complexity of k-means ++ is proportional to the log of $K$ and approximately linear with respect to the number of agents (Arthur and Vassilvitskii 2007). In our adaptation of k-means++, we begin by selecting an agent at random to initialize the first subcoalition and define the subcoalition's collective weak order according to that agent's preferences. Then for every subsequent subcoalition to be initialized, we select a new agent, where the probability of being selected is inversely proportional to that agent's utility with respect to the subcoalitions that have already been seeded with an agent. Once all subcoalitions have been seeded, we derive the collective weak order for each subcoalition using the $I_{2}$ rule and then assign agents to the subcoalition whose collective weak order maximizes their utility.

The following pseudo-code defines the initialization step in the sCCA algorithm:

1. Choose an initial agent $i$ uniformly at random from $N$. Let $r_{1}=f\left(V_{i}\right)$.

2. Choose the next agent $i^{\prime}$, selecting $i^{\prime} \in N$ with probability $\frac{\min \left(u\left(V_{i^{\prime}}, r_{1}\right), \ldots, u\left(V_{i^{\prime}}, r_{k-1}\right)\right)}{\sum_{i \in N} \min \left(u\left(V_{i}, r_{1}\right), \ldots, u\left(V_{i}, r_{k-1}\right)\right)}$. Let $r_{k}=f\left(V_{i^{\prime}}\right)$.

3. Repeat step 2 until $K$ initial agents have been chosen. Let $\pi_{0}$ represent a partition of these agents into $K$ subcoalitions with each subcoalition containing one agent.

4. For each initialized subcoalition, calculate $f\left(\mathbf{V}_{k}\left(\pi_{0}\right)\right)$, which is the initialized set of collective weak orders given the singleton subcoalitions.

5. Assign each agent $i \in N$ to the collective weak order that maximizes their utility, which defines the initial partition $\pi_{1}$ of individuals into the set of subcoalitions $S_{k}\left(\pi_{1}\right)$.

3.4.4. Body: The updating step After initializing the subcoalition structure, the sCCA algorithm begins the search for a weakly stable partition. Following the initialization step, each subcoalition's collective weak order is optimal for a single agent, but the subcoalitions are populated by many agents with diverse preferences. The updating step thus involves updating the collective weak orders for each subcoalition so that they maximize the aggregate utility of their respective memberships. 
Let $t$ index iterations of the updating-and-assignment process. The updating step inherits a partition of individuals $\pi_{t}$ which define a set of subcoalitions $S_{k}\left(\pi_{t}\right)$, and an associated set of collective weak orders $f\left(\mathbf{V}_{k}\left(\pi_{t-1}\right)\right)$. It returns $f\left(\mathbf{V}_{k}\left(\pi_{t}\right)\right)$, the updated set of collective weak orders that are consistent with $\pi_{t}$.

3.4.5. Body: The assignment step Following the updating step, however, some agents may no longer be in subcoalitions that maximize their utility. If any such agents exist, the assignment step reassigns these agents to the subcoalitions whose collective weak orders are strictly more desirable than the collective weak order of the subcoalition to which they are currently assigned.

The assignment step inherits a partition $\pi_{t}$, a set of subcoalitions $S_{k}\left(\pi_{t}\right)$, and a set of collective weak orders $f\left(\mathbf{V}_{k}\left(\pi_{t}\right)\right)$. It returns $\pi_{t+1}$ and $S_{k}\left(\pi_{t+1}\right)$, which are a partition and set of subcoalitions for which all agents are in a subcoalition whose collective weak order maximizes their utility.

3.4.6. Body: Algorithm termination The updating-and-assignment steps continue until the partitions in two consecutive iterations are identical, which means that the partition is weakly stable. This partition is thus returned as $\pi_{K}^{*}$.

3.4.7. Inner loop: Searching for high-quality subcoalitions Our concern that the body of sCCA might discover a low-quality partition by chance lead us to recommend replicating the body of sCCA multiple times. Thus, the inner loop returns the $\pi_{K}^{*}$ that has the highest aggregate utility after $B$ replications of the body, denoted $\hat{\pi}_{K}^{*}$. In cases where the analyst exogenously determines the number of subcoalitions in the data, $\hat{\pi}_{K}^{*}$ is the partition returned by sCCA.

3.4.8. Outer loop \& $K^{*}$ function: Identifying $K^{*}$ In many cases, the analyst may not know $K^{*}$ ex ante and may instead desire to estimate it from the data given candidate values of $K$. Therefore, we next describe a set of methods that can assist in determining $K^{*}$ conditional on having calculated $\hat{\pi}_{K}^{*}$ for varying $K$.

One way to estimate $K^{*}$ involves calculating $\hat{\pi}_{K}^{*}$ for each $K$ and then returning the aggregate utility for each. Comparisons of aggregate utility given $\hat{\pi}_{K}^{*}$, which we represent as $u\left(\hat{\pi}_{K}^{*}\right)$, inform how much better off agents are when $K$ increases. If $u\left(\hat{\pi}_{K}^{*}\right)$ increases meaningfully given a one unit increase in $K$, it implies that partitions with one additional subcoalition make agents much better off and thus that the data are more consistent with the higher $K$. However, if $u\left(\hat{\pi}_{K}^{*}\right)$ increases only slightly, this instead implies that agents are roughly indifferent between the two partitions and that the data are instead consistent with the lower $K$.

But, how can we know what constitutes a meaningful difference? Two methods that are commonly used to evaluate the optimal $K$ for the $k$-means clustering algorithm can also be applied 
here. One is to identify a $K$ such that $u\left(\hat{\pi}_{K}^{*}\right)-u\left(\hat{\pi}_{K-1}^{*}\right)>>u\left(\hat{\pi}_{K+1}^{*}\right)-u\left(\hat{\pi}_{K}^{*}\right)$. This is often referred to as an "elbow" in the graph relating $K$ to $u\left(\hat{\pi}_{K}^{*}\right)$. The logic behind the elbow test is that, in the presence of unclustered data, the slope of the curve relating $K$ to $u\left(\hat{\pi}_{K}^{*}\right)$ should be constant (Tibshirani et al. 2001).

The elbow test version of the $K^{*}$ function involves the following steps:

1. For each $K, K+1 \in \mathcal{K}$, calculate $\delta_{K}=u\left(\hat{\pi}_{K+1}^{*}\right)-u\left(\hat{\pi}_{K}^{*}\right)$.

2. Return the minimum $K$ such that $\delta_{K}>>\delta_{K+1}$.

However, there may be ambiguity regarding which $K$ constitutes the elbow or whether an elbow exists at all. Further, the assumption that $u\left(\hat{\pi}_{K}^{*}\right)$ declines with a constant slope in the presence of unclustered data may not be valid for a given dataset. A more robust version of the elbow test, called the gap statistic, addresses these shortcomings. The gap statistic version of the elbow test simulates $\hat{\pi}_{K}^{*}$ as a function of $K$ based on synthetic, unclustered data. It then uses this null distribution of partitions in the absence of subcoalitions $\hat{\boldsymbol{\pi}}_{K}^{0}$ as a control when executing the elbow test (Tibshirani et al. 2001).

When compared to the elbow test, the gap statistic has two advantages. The first advantage is that the gap statistic gives the analyst confidence that the observed elbow would not be so observed if no subcoalitions existed. A second advantage is that we can use the distribution of $\hat{\boldsymbol{\pi}}_{K}^{0}$ to develop tests for identifying $K^{*}$ that take into account not only the slope of the function relating $K$ to $u\left(\hat{\pi}_{K}^{*}\right)$, but also its variance. We recommend selecting the $K$ such that the gap statistic for $K$ is at least two standard deviations from the mean above the gap statistic for $K+1$.

Our implementation of the gap statistic is as follows:

1. For $b_{0}$ in $1, \ldots, B_{0}$ :

a. Construct a synthetic set of votes by randomly permuting the identity of the agents associated with votes in the original data. Specifically, for every vote $v_{i, a, a^{\prime}}$ in the data, create a synthetic vote $v_{i^{\prime}, a, a^{\prime}}$ where $i^{\prime}$ is sampled with replacement from $N$ with weight equal to the number of votes over alternatives recorded by agent $i$. Define $V_{i^{\prime}, b_{0}}$ as the multiset union over all votes for synthetic agent $i^{\prime}$.

b. For all $K \in \mathcal{K}$, calculate $u\left(\hat{\pi}_{K, b_{0}}^{0}\right)$ based upon $\hat{\pi}_{K, b_{0}}^{0}$, where $\hat{\pi}_{K, b_{0}}^{0}$ is the weakly stable partition defined for the synthetic data.

c. For all $K \in \mathcal{K}$, return $u^{\prime}\left(\hat{\pi}_{K, b_{0}}^{*}\right)=u\left(\hat{\pi}_{K, b_{0}}^{0}\right)-u\left(\hat{\pi}_{K}^{*}\right)$.

2. For all $K \in \mathcal{K}$, estimate $E\left[u_{N}^{\prime}\left(\hat{\pi}_{K}^{*}\right)\right]=B_{0}^{-1} \sum_{b_{0}=1}^{B_{0}} u_{N}^{\prime}\left(\hat{\pi}_{K, b_{0}}^{*}\right)$ and

$$
s_{K}=\left(B_{0}-1\right) \sum_{b_{0}=1}^{B_{0}}\left(E\left[u_{N}^{\prime}\left(\hat{\pi}_{K}^{*}\right)\right]-u_{N}^{\prime}\left(\hat{\pi}_{K, b_{0}}^{*}\right)\right)^{2}
$$

3. Return the minimum $K$ such that $E\left[u_{N}^{\prime}\left(\hat{\pi}_{K}^{*}\right)\right] \geq E\left[u_{N}^{\prime}\left(\hat{\pi}_{K+1}^{*}\right)\right]+2 s_{K+1}$. 
A different approach for identifying $K^{*}$ involves calculating the clustering stability of the partition rather than the aggregate utility. Clustering stability-based evaluation of a partition is based on the theory that meaningful partitions should be robust to small perturbations in the underlying data caused by sampling variability (von Luxburg 2010). If there are more subcoalitions estimated than actually exist in the data, small changes to the set of agents resulting from different samples will tend to produce substantially different partitions of agents. Note that clustering stability differs from prior concepts of stability introduced in the paper. The former requires that the partition is robust to different sets of agents. The latter requires that the partition be robust to the presence of goal-oriented or (boundedly-)rational agents.

A straightforward way to test the clustering stability of a partition is to repeatedly resample agents (with replacement), reestimate the partitions from the resampled data, and then calculate the average proportion of agents who fall in the same subcoalition in the resampled data as in the real data. If large shares of agents fall in the same subcoalitions in both the synthetic and real data, it is indicative of meaningful subcoalitions. Similar to the elbow test, interpreting the mapping from $K$ to average stability in order to determine $K^{*}$ requires judgment by the analyst regarding when the clustering stability level is deemed unacceptably low. When the elbow test, gap statistic, and clustering stability-based approaches all agree, however, it provides strong evidence for a given $K^{*}$.

Our recommended clustering stability-based test for $K^{*}$ is implemented as follows:

1. For $b_{0}$ in $1, \ldots, B_{0}$ :

a. Construct a synthetic set of agents $i^{\prime} \in N_{0}$ by resampling uniformly from $N$ with replacement. Define $V_{i^{\prime}, b_{0}}$ as the multiset union over all votes for synthetic agent $i^{\prime}$.

b. For all $K \in \mathcal{K}$, compute $\tilde{\pi}_{K, b_{0}}^{*}$, which is the weakly stable partition defined for the synthetic data.

c. Define two partitions $\pi, \pi^{\prime}$ as equivalent if for all agents $i, j \in N$ and for all $i^{\prime}, j^{\prime} \in N^{\prime}$ if $V_{i}=V_{i^{\prime}}, V_{j}=V_{j^{\prime}}$ and $i, j \in S_{k}(\pi)$ for some $k \in\{1, \ldots, K\}$ then $i^{\prime}, j^{\prime} \in S_{k}\left(\pi^{\prime}\right)$ for some $k \in$ $\{1, \ldots, K\}$. Define the minimum matching distance between two partitions as the minimum number of agents who must be moved to a new subcoalition for $\tilde{\pi}_{K, b_{0}}^{*}$ to be equivalent to $\hat{\pi}_{K}^{*}$. For all $K \in \mathcal{K}$, return the minimum matching distance between $\tilde{\pi}_{K, b_{0}}^{*}$ and $\hat{\pi}_{K}^{*}$, denoted $\Delta_{K}\left(\tilde{\pi}_{K, b_{0}}^{*}, \hat{\pi}_{K}^{*}\right)$.

2. For all $K \in \mathcal{K}$, calculate the clustering stability score:

$$
\bar{\Delta}_{K}=\frac{1}{\left|N_{0}\right|} B_{0}^{-1} \sum_{b_{0}=1}^{B_{0}} \Delta_{K}\left(\tilde{\pi}_{K, b_{0}}^{*}, \hat{\pi}_{K}^{*}\right)
$$

3. Return the minimum $K$ such that $\Delta_{K}>>\Delta_{K+1}$. 
When these three methods identify the same number of subcoalitions as fitting the data bestand in our experience they often do - the analyst should feel confident that the returned partition captures the latent structure of conflict in the organization. In cases where they disagree, it is up to the analyst to determine whether changes to aggregate utility or changes to clustering stability more meaningfully identify subcoalitions in a given empirical setting. When the results of these tests are ambiguous, we generally recommend choosing the partition associated with fewer subcoalitions rather than more subcoalitions, which is based on our preference for a more parsimonious model, all else being equal.

\section{Applying sCCA to Three Cases}

We next apply sCCA to three cases. The primary purpose of the cases is to demonstrate the range of research questions to which sCCA can be applied and, in the process, types of data that can be analyzed by sCCA. Each case analysis is built around a question faced by a manager or community leader. The first case uses sCCA to replicate an attempt by the editors of the organizational sociology blog OrgTheory.net to determine whether their community-based ranking of sociology departments was biased by a small cadre of unrepresentative voters. The second uses sCCA to model the structure of internal conflict over the future of Wikipedia based on a survey of editors that elicited recommendations for improving the platform. The third uses sCCA to understand the the emergence of intragroup conflict among writers electing candidates for the National Baseball Hall of Fame and to evaluate whether or not social influence processes made the schism self-reinforcing.

\subsection{Case 1: A "Fabio Effect" in the OrgTheory.net 2013 Sociology Department Ranking Survey}

4.1.1. Summary Shortly after U.S. News and World Report published its 2013 rankings of sociology departments, editors at the popular OrgTheory.net blog administered a survey of readers to derive a crowdsourced ranking that would better represent the sentiment of the broader sociology community. The poll was conducted on the All Our Ideas platform, an open-source platform for conducting Wiki surveys designed for voters to make "as many head-to-head snap judgments as possible" (Healy 2013). In the survey, participants were randomly presented with head-to-head matchups between sociology departments one at a time, with the prompt "In your judgment, which of the following is the better Sociology department?" Respondents were free to evaluate as many pairwise contests as they desired. Around 75 percent voted in 70 or fewer contests, with a small number registering upwards of 500 votes. In all, 635 participants voted 46,317 times across the 19,460 possible matchups of the 140 departments.

The All Our Ideas platform estimated a hierarchical probit model to rank departments based on the assumption that the probability that one department defeats another in a pairwise comparison 
is a function of the relative appeal of each department (for more detail, see Salganik and Levy 2015). The parameter values returned by the regression model ranked UC-Berkeley, University of Michigan, Harvard University, Princeton University, and University of Chicago most highly. However, it came as a surprise to the editors of the blog that Indiana University was ranked as a top-10 department, which led Kieran Healy, the organizer of the survey, to playfully speculate that "Indiana's high ranking is due solely to Fabio mobilizing a team of role-playing enthusiasts to relentlessly vote in the survey" (Healy 2013), referring to Fabio Rojas, a sociology professor at Indiana University and frequent contributor to the blog.

In a subsequent blog post, Healy reanalyzed the data in order to test the hypothesis of the "Fabio effect." Healy reasoned that "supervoters" who persevered through many, many head-tohead contests were likely to be unrepresentative of the broader population and re-estimated the rankings after excluding them. Healy found that Indiana University dropped from $9^{\text {th }}$ to $12^{\text {th }}$, which was suggestive evidence in favor of the hypothesis. Healy also noted that one supervoter favored the University of Notre Dame, perhaps inflating its ranking as well.

The problem of determining whether there indeed exists a subset of voters with preferences that are distinct from the majority view is one useful application of sCCA. Further, Wiki surveys like this are an excellent example of the flexibility of sCCA to estimate partitions of voters based on incomplete data, since even the most devoted participants in the survey only evaluated a small fraction of the possible head-to-head comparisons.

4.1.2. Data The data, which we download from the All Our Ideas website, is a spreadsheet in which each row records a participant ID and the results of each head-to-head comparison. We then encode the data in an adjacency matrix for each participant for which each row and column represents one of the sociology departments being evaluated. A cell in the adjacency matrix is assigned a "1" if the department associated with the row is preferred to the department associated with the column in a given head-to-head comparison. All other cells are marked "0." This simple data structure thus permits all four possible relationships between departments (either department wins, they tie, or no preferences are expressed) and also permits voters to have intransitive preferences over departments.

4.1.3. Results We then use sCCA to evaluate the "Fabio effect" hypothesis. Specifically, we examine the collective weak order of sociology departments in the absence of subcoalitions and then evaluate whether they change in the presence of two subcoalitions.

When the $I_{2}$ rule is applied to all of the voters, there are 11 departments that tie for the highest rank: Harvard University, Indiana University, Princeton University, Stanford University, UC-Berkeley, UCLA, UNC-Chapel Hill, University of Chicago, University of Michigan, University 
of Pennsylvania, and University of Wisconsin. These are the same as the top 11 returned by the All Our Ideas platform.

Next, we apply the sCCA algorithm, setting the number of subcoalitions $K=2$. We find that 571 individuals are assigned to the first subcoalition, and 64 to the second. Notwithstanding the relatively modest size of the second subcoalition, this partition is Nash stable. In the first subcoalition's collective weak order, Princeton University and UC-Berkeley are tied for the top rank, followed by a 10-way tie among Columbia University, Harvard University, Northwestern University, Stanford University, UCLA, UNC-Chapel Hill, University of Chicago, University of Michigan, University of Pennsylvania, and University of Wisconsin. Indiana University is ranked as part of a 17-way tie in the next-lowest tier of departments. However, in the second subcoalition, University of Indiana is ranked in the first tier, tied with Case Western University, Mississippi State University, University of Alberta, and University of Notre Dame. The evaluations of these 64 participants thus clearly departed from those of the majority and in a manner that inflated Indiana University's relative ranking when the data was analyzed collectively: $:^{2}$

While an informal ranking of sociology departments by followers of a popular blog is perhaps not the most consequential conflict over collective outcomes, it highlights important features of sCCA. First, it is telling that Healy, a sociologist whose research focuses on the "impact of models and measurement on social classification" 3 lacked a computational method for partitioning voters in order to produce competing departmental rankings across multiple subgroups. Second, the case emphasizes how important it is for a method to permit incompleteness in observational data. If the survey had required complete comparisons over alternatives, then each respondent would have had to make nearly 20 thousand comparisons between departments. This could have been simplified, of course, by asking participants to rank-order departments. But, in that case, voters would be required to have complete, transitive preferences, making fine-grained comparisons between departments they perhaps know little about. Finally, this case emphasizes why computational efficiency considerations are paramount in the design of an algorithm seeking to identify stable partitions in data. With 635 participants and 140 departments, there were over 400 thousand possible partitions dividing participants into two subcoalitions and approximately $1.9 \times 10^{263}$ possible collective weak orders for each subcoalition. Enumerating all possible partitions and all possible collective weak orders for each partition would clearly not be computationally feasible.

\footnotetext{
${ }^{2}$ It is worth pointing out that Notre Dame was the home university of frequent blog contributor Omar Lizardo. So, perhaps, Healy would have more accurately named this phenomenon the "Fabiomar effect."

${ }^{3}$ Excerpted from his personal website, https://kieranhealy.org/
} 


\subsection{Case 2: Wikipedia's Editor Rebellion}

4.2.1. Summary Next, we turn to a more consequential intraorganizational conflict that emerged at Wikipedia in the early 2010s. Following rapid growth in editor participation from 2003 to 2007, the number of new editors active on Wikipedia suddenly began to decline (Suh et al. 2009). Wikipedia responded with new tools and infrastructure designed to make editing easier, but with little impact. In his 2009 "State of the Wiki" address, founder Jimmy Wales argued that "if active contributors continue to decrease, there may not be a large enough cohort to 'look after' Wikipedia" (Wales 2009). Concerns that a smaller, more homogeneous editor population would jeopardize the future health of the Wikipedia project prompted a focus on editor recruitment and retention in the Wikimedia Foundation's 2011-2015 strategic plan. As part of this effort, Wikipedia designed a series of surveys in order to better understand the experience of editors and recommend strategic changes.

Inside Wikipedia, there were competing theories about the causes of the weakening editor participation. The first theory was that editing Wikipedia articles was too technically demanding. By creating an easier user interface for editors, more people would be attracted to the community. The second theory was that the culture was hostile to new editors. Rather than welcoming new users and encouraging them to learn the norms of the platform, experienced editors would delete the edits of new editors that did not conform with Wikipedia's guidelines without providing constructive feedback. The editor survey offered the potential to gather anonymous feedback from users in order to prioritize changes to the platform and community standards.

4.2.2. Data We analyze a question from the 2012 Wikipedia Editor Survey in which respondents were asked to identify "the most important problems that have affected you personally, making it harder for you to edit." Editors were given a list of ten potential problems and had the opportunity to select up to three they found most relevant to their experience. We encoded cases in which one problem was deemed relevant and the other problem not deemed relevant as a vote for the former over the latter. This analysis therefore also takes advantage of sCCA's ability to accept incomplete data, because we do not record votes for problems that are either both deemed as relevant or both not deemed as relevant.

In Wikipedia's topline analysis, approximately two-fifths of the over 17 thousand participants surveyed criticized "editors who feel like they 'own' specific articles and don't want others to contribute to them." Thirty-two percent felt that there were "too many rules and policies." Between twenty and thirty percent responded affirmatively to four of the remaining eight options. The results supported the theory that Wikipedia's editorial culture was its biggest problem, but that the other concerns were valid as well. 
4.2.3. Results We use sCCA to model the internal disagreement over Wikipedia's most important problems. We restrict the data set to editors who contribute primarily to English Wikipedia and who indicate that they at least sometimes participate as editors. After excluding editors who selected none of the problems, we are left with 1,416 individuals in our dataset.

We present the collectively-defined relative importance of problems conditional on the number of subcoalitions in Figure 2. When analyzed as a single group, our results are consistent with the results of Wikipedia's topline analysis. The biggest problem is editors who "feel like they own specific articles." Tied are "too many rules and policies," the editing interface being "hard to use," editors not being "fun to work with," "lack of support from other editors," "lack of access to research materials," and "criticism of you and your work." Lowest ranked were problems related to the software, "warning messages on your talk page," and "harassment" by other editors.

However, when we analyze Wikipedia as two subcoalitions, we identify substantial internal conflict. The first subcoalition ("A"), which includes 726 editors, points to the number of rules, difficulties using the editing interface, and lack of access to research materials as the most important problems. The other subcoalition ("B"), which includes 690 editors, instead identifies editorial ownership of articles, editors not being fun to work with, and criticism of one's work as the most serious problems. Strikingly, two of the problems that were most important to the former group were least important to the latter.

The topline analysis thus obscures an important division among editors over perceived problems with the platform. Subcoalition A identifies technical problems as the most important problems for Wikipedia to address. Subcoalition B identifies cultural problems as more important. This divide is also reflected in the difference between the average utility of editors with the preferences of their own subcoalition - which are -8.3 and -8.8 for subcoalitions A and B, respectively-against the average utility of editors with the preferences of the other subcoalition - which are -21.6 and -23.8. When we instead allocate the editors among three subcoalitions, the third's hierarchy of problems is mix of the two identified previously. Their top problem is a lack of access to research materials, but their second tier of problems includes both issues with other editors and difficulties using the editing platform. For four subcoalitions, similarly, one group continues to emphasize cultural issues, one emphasizes technical ones, and the other two reflect a blend of priorities.

Next, we use the three methods described in subsubsection 3.4.8 to identify the number of subcoalitions in the editor population from the data. The left panel of Figure 3 reports aggregate utility levels conditional on subcoalition count for partitions with one through five subcoalitions. The "elbow" that appears at two subcoalitions suggests that a meaningful second subcoalition may indeed be present. The center panel reports the gap statistic - i.e., the difference between the aggregate utility when individual preferences are randomized in 100 synthetic datasets and the aggregate 
Figure 2 Wikipedia: Aggregate Group and Subcoalition Preferences

\begin{tabular}{|l|c|}
\hline Aggregate Group Preferences & Rank \\
\hline Editors who feel like they own specific articles and don't want others to contribute to them & 3 \\
\hline Too many rules and policies & 2 \\
The editing interface is hard to use & Editors who are not fun to work with \\
Lack of support from other editors \\
Lack of access to research materials like scholarly articles and books \\
Criticism of you and your work, including reverts and deletions & 1 \\
\hline $\begin{array}{l}\text { The software for doing maintenance tasks (such as patrolling and fighting vandalism or spam) is too hard to use } \\
\text { Warning messages on your talk page } \\
\text { Harassment by other editors }\end{array}$ & \\
\hline
\end{tabular}

\begin{tabular}{|l|l|}
\hline 2-Group Top Preferences: Subcoalition A & 2-Group Top Preferences: Subcoalition B \\
\hline $\begin{array}{l}\text { Too many rules and policies } \\
\text { The editing interface is hard to use } \\
\text { Lack of access to research materials like scholarly articles and books }\end{array}$ & $\begin{array}{l}\text { Editors who feel like they own specific articles and don't want others to } \\
\text { contribute to them } \\
\text { Editors who are not fun to work with } \\
\text { Criticism of you and your work, including reverts and deletions }\end{array}$ \\
\hline 2-Group Bottom Preferences: Subcoalition A & 2-Group Bottom Preferences: Subcoalition B \\
\hline Harassment by other editors & $\begin{array}{l}\text { The editing interface is hard to use } \\
\text { The software for doing maintenance tasks (such as patrolling and fighting } \\
\text { vandalism or spam) is too hard to use } \\
\text { Lack of access to research materials like scholarly articles and books }\end{array}$ \\
\hline
\end{tabular}

\begin{tabular}{|l|l|l|}
\hline $\begin{array}{l}\text { 3-Group Top Preferences: } \\
\text { Subcoalition A }\end{array}$ & $\begin{array}{l}\text { 3-Group Top Preferences: } \\
\text { Subcoalition B }\end{array}$ & $\begin{array}{l}\text { 3-Group Top Preferences: } \\
\text { Subcoalition C }\end{array}$ \\
\hline $\begin{array}{l}\text { Too many rules and policies } \\
\text { The editing interface is hard to use }\end{array}$ & $\begin{array}{l}\text { Editors who feel like they own specific articles } \\
\text { and don't want others to contribute to them } \\
\text { Editors who are not fun to work with } \\
\text { Criticism of you and your work, including reverts } \\
\text { and deletions }\end{array}$ & $\begin{array}{l}\text { Lack of access to research materials like } \\
\text { scholarly articles and books }\end{array}$ \\
\hline $\begin{array}{l}\text { 3-Group Bottom Preferences: } \\
\text { Subcoalition A }\end{array}$ & $\begin{array}{l}\text { 3-Group Bottom Preferences: } \\
\text { Subcoalition B }\end{array}$ & $\begin{array}{l}\text { 3-Group Bottom Preferences: } \\
\text { Subcoalition C }\end{array}$ \\
\hline $\begin{array}{l}\text { Lack of support from other editors } \\
\text { Lack of access to research materials like } \\
\text { scholarly articles and books }\end{array}$ & The editing interface is hard to use & Harassment by other editors \\
\hline
\end{tabular}

utility in the observed data-along with 95 percent confidence intervals. The gap statistic for two subcoalitions is significantly lower than when the editors are treated as a single group. In contrast, moving from two subcoalitions to three subcoalitions does not change the gap statistic significantly. The right panel reports the clustering stability score across 100 bootstrapped datasets. The score remains as high as 0.9 for two subcoalitions, dropping greatly if a third is added. All three of the methods therefore agree that the population of editors is well-characterized by two subcoalitions. 
The two-subcoalition partition also satisfies Nash stability, offering additional confidence that the identified subcoalitions faithfully reflect the structure of conflict inside Wikipedia.

Figure 3 Wikipedia: Utility, Gap Statistic, and Clustering Stability Score by Number of Subcoalitions Utility Gap Statistic
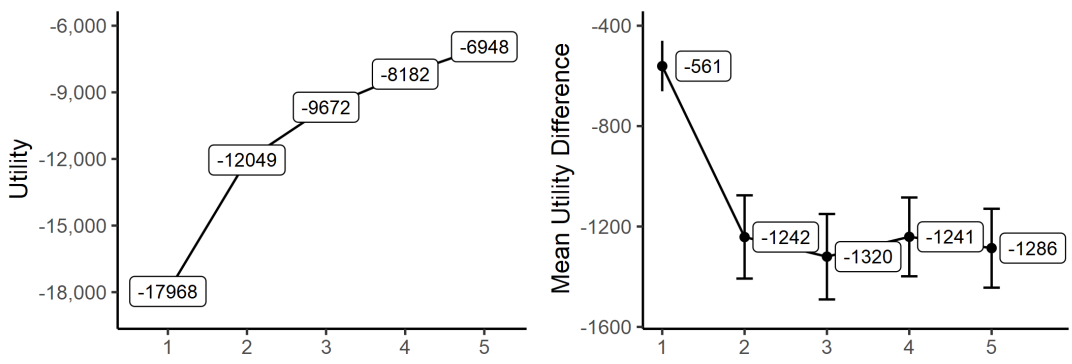
Clustering Stability Score

Number of Subcoalitions

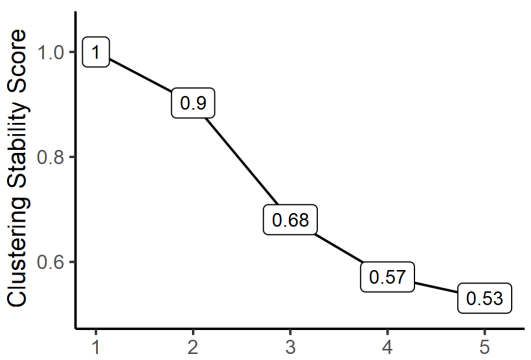

We next examine what types of editors are members of each subcoalition. We use logistic regression to predict membership in subcoalition $\mathrm{B}$ conditioned on the amount of time spent on Wikipedia, editorial experience, age, gender, and country of origin. We find that editors who spent more than an hour in the last week contributing to Wikipedia are 16 percent more likely to be a member of subcoalition $\mathrm{B}(\beta=0.067, s e=0.012)$. Further, each additional year of editing experience makes an editor three percent more likely to be a member of subcoalition $\mathrm{B}(\beta=0.11$, $s e=0.022)$. Both effects are significant at the 0.01-confidence level. Gender, age, and countryof-origin are not significant predictors of subcoalition membership. Experienced editors and avid users are more likely to be a member of the subcoalition who believes that other editors are the most significant problem with Wikipedia. In contrast, inexperienced and less frequent users are more likely to point to the problems associated with the editing platform itself.

This conflict between the subcoalitions erupted when English Wikipedia introduced its new Visual Editor in 2013 in order to make it easier for new editors on the site. Existing editors complained that the bug-marred rollout was disrespectful to dedicated users, a waste of donated money, and that it reflected a lack of commitment to Wikipedia's core mission. In response, editors "staged a rebellion" by adopting user-written code that allowed them to circumvent the new editing platform, leading Wikipedia to grudgingly change the Visual Editor interface from opt-out to optin (Orlowski 2013, Sampson 2013). Had leadership been aware that the rationale for this major change to the editing interface was in conflict with the desires of its most avid users, they would also have known they needed to be more careful during the Visual Editor's introduction.

This case emphasizes how excessive attention to aggregated preferences or topline survey results may lead managers to overlook the potential for conflict over important decisions. Because 
Wikipedia's leadership lacked insight on the structure of conflict within the organization, they could not appreciate the sharp divergence in preferences across the two subcoalitions and, as a result, underestimated the internal resistance to a strategic change. The case also shows how the information returned by sCCA about subcoalition membership and preferences can be used to understand the major sources of intraorganizational conflict and the correlates of subcoalition membership. Moreover, this case reiterates how sCCA can be applied in the presence of sparse data and demonstrates the application of algorithms for identifying the number of subcoalitions in the data.

\subsection{Case 3: The Base Ball Writers' Association of America Tosses a Shutout}

4.3.1. Summary The most important public function of the Base Ball Writers' Association of America (BBWAA) is determining which retired baseball players are inducted into the National Baseball Hall of Fame. Beginning in 2013, however, the election process became embroiled in controversy when none of the candidates received the seventy-five percent of votes required for induction. For the first time in a half-century, the National Baseball Hall of Fame had a summer induction ceremony with no living inductees. The BBWAA considers Hall of Fame voting to be "the ultimate privilege" for its members (Chappell 2014). Yet rumors swirled that, if the BBWAA continued to be unable to serve its purpose of selecting candidates for the Hall of Fame, the Hall of Fame would begin to explore other options for determining who should be inducted (Keri 2013).

Prior to the election, baseball writers acknowledged that 2013's crop of new candidates were particularly controversial. Barry Bonds and Roger Clemens, two players who are among the most distinguished in the game's history but whose candidacies were shrouded in rumors of performanceenhancing drug (PED) use, were submitted to the baseball writers for consideration for the first time. Absent the PED question, both Bonds and Clemens would be near-consensus Hall of Fame picks. While other marginal Hall of Fame candidates suspected of PED use had been considered by the writers in prior years, this was the first time they had to judge players with such extraordinary on-the-field performance who were also suspected of using PEDs. Despite many baseball writers acknowledging a crowded, controversial ballot with many qualified newcomers, few suspected that no candidates would be inducted.

New York Times columnist Nate Silver hypothesized that the tenor of the debate had changed during the 2013 voting process: "Instead of the typical friendly arguments about how a player's lifetime accomplishments might be weighed against how dominant he was in his best seasons, or how to compare players at different positions, the writers are now spending most of their time arguing about who used steroids and when, and how this should affect Hall of Fame consideration" (Silver 2013). Disagreements over players prior to 2013 tended to be idiosyncratic, as each voter 
applied slightly different criteria for measuring greatness. The 2013 disagreement, instead, felt more like competing factions within the BBWAA: the writers who felt that PED rumors disqualified a player from Hall of Fame induction against those willing to weigh possible PED use against on-the-field performance.

4.3.2. Data Many baseball writers voluntarily make their Hall of Fame ballots public. Beginning with the 2009 class of candidates, Twitter user @leokitty began tracking these public votes. In 2013, Ryan Thibodeaux took over the ballot tracking effort in a public spreadsheet posted on his website. In addition, starting in 2013, the BBWAA began to publish the votes of willing writers. Over time, the number of public ballots has increased, from 60 in 2009, to 168 in 2013, to 311 in 2016.

We use these public ballots to study the nature and evolution of the conflict among the baseball writers over Hall of Fame candidates. From each writer's ballot, we create a series of binary comparisons between players, assuming that writers prefer players for whom they voted to those for whom they did not. We do not record binary preferences over candidates when a writer either voted for both candidates or excluded both candidates from their ballot.

4.3.3. Results We analyze whether the nature of disagreement over which candidates deserve induction into the Hall of Fame changed in 2013, the first year that Bonds and Clemens were on the Hall of Fame ballot. Then, we examine whether subcoalition conflict in the BBWAA has changed how voters use other writers' prior votes to update their beliefs about which candidates to vote for in future years.

We begin by examining the aggregate utility conditional on the number of subcoalitions for each year from 2009 through 2016, displayed in the first column of Figure 4. Because there are different numbers of writers and candidates in each year, we scale utility so that it equals -1 when the writers are treated as a single group. The longitudinal data identify a clear elbow beginning with the 2013 ballot that did not exist in the earlier years. From 2009 through 2012, the relationship between the number of subcoalitions and aggregate utility is roughly linear. From 2013 through the present, however, it is highly nonlinear, with an elbow at two groups. This supports the hypothesis that there is one subcoalition from 2009 through 2012, but two distinct subcoalitions from 2013 on.

The results from the 2013 election are illustrative of the nature of the conflict. When the writers are treated as a single bloc, Clemens and Bonds are ranked in the second-highest tier behind five other candidates with no ties to PED-use. When writers are divided into two subcoalitions, however, writers are nearly evenly split between a PED-sensitive subcoalition that ranks Bonds and 
Figure 4 BBWAA: Aggregate Utility, Gap Statistic, and Clustering Stability Score by Number of Subcoalitions
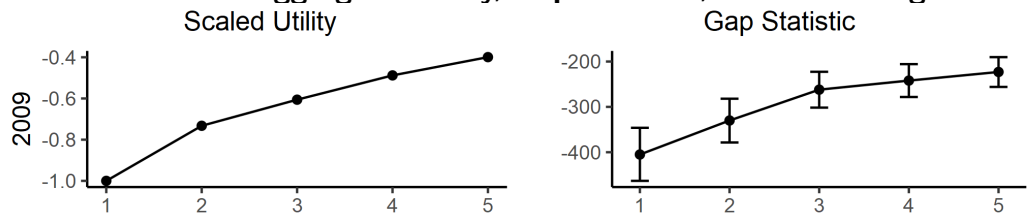
Clustering Stability Score
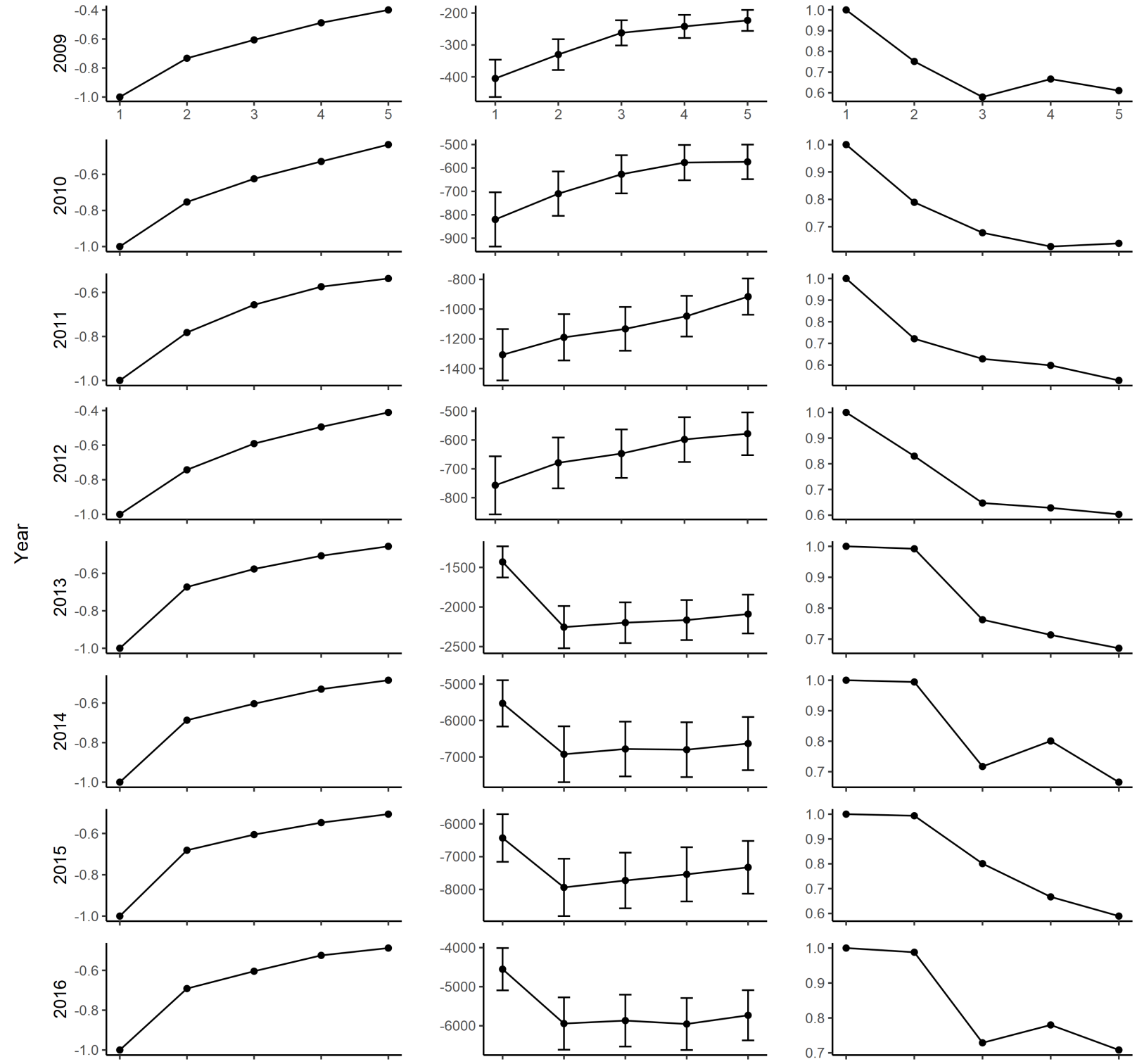

Number of Subcoalitions

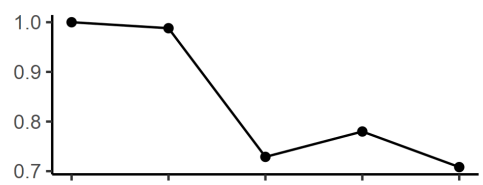

Clemens as tied for last place and a PED-insensitive coalition that ranks Bonds first and Clemens second, ahead of all other candidates.

The second column of Figure 4 reports the gap statistic, which also indicates a clear shift beginning in 2013. Prior to 2013, the gap statistic is monotonically increasing with respect to the number of subcoalitions. After 2013, it is lower for two subcoalitions than for one, then remains roughly constant when we partition the writers into 3 , 4 , or 5 subcoalitions. 
The third column of Figure 4 reports the clustering stability score. Again, the pattern changes after 2013. Prior to 2013, when the writers are divided into two subcoalitions, many voters who are identified as a part of the same subcoalition in the observed data are part of different subcoalitions in the bootstrap samples. After 2013, the subcoalitions produced by the bootstrap samples almost exactly align with those produced by the actual data when the writers are divided into two subcoalitions. Once again, the three methods agree that the BBWAA becomes well-characterized as two competing subcoalitions after 2013. And, as in the previous cases, the two-subcoalition partitions post-2013 satisfy Nash stability.

We next use these results to examine whether changes to patterns of social influence are contributing to the ossification of subcoalition boundaries and subsequent difficulty reaching consensus on candidates. Baseball writers frequently cite each other's analysis and prior voting behavior when publicly justifying their voting choices, creating a bandwagon effect for some candidates. Although some candidates are elected in their first year on the ballot, most face a slower process through which writers gradually become convinced of a candidate's merit following many years of debate, balloting, and re-evaluation (James 1994). This multiyear conversation about whether a player is worthy of induction has tended to generate coordination among the electorate. As a result, every player but one who has ever received 50 percent of the vote from the writers has eventually been inducted.

However, if baseball writers' evaluations of candidates are influenced more strongly by writers in their own subcoalition than by writers outside their subcoalition, then the social influence of the out-group writers would be weakened and the cross-subcoalition divergence in evaluation of the candidates would be self-reinforcing. Goldberg and Stein (2018) refer to this mechanism for explaining self-reinforcing conflict as associative diffusion. In contrast, if baseball writers are influenced equally by members of their own subcoalition as by members of the opposing one, then the divergence would be more likely to weaken over time.

We examine these dynamics of social influence among the writers using a series of regression analyses. These models estimate whether a writer, $i$, voted for a candidate $j$ in year $t$, where a vote by a writer for a candidate in a year, $v_{i j t}$, is represented by a 1 and the absence of a vote is represented by a 0 . In all years, we divide the writers into two subcoalitions. The inclusion of a lagged dependent variable complicates estimation of discrete choice models Honore and Kyriazidou 2000), so we estimate linear probability models of the following form:

$$
v_{i, j, t+1}=\delta v_{i, j, t}+\beta_{1} E\left(v_{i, j, t} \mid \text { in subcoalition }\right)+\beta_{2} E\left(v_{i, j, t} \mid \text { out of subcoalition }\right)+\lambda_{t}+\epsilon_{i, j, t}
$$

$\beta_{1}$ measures the influence of in-subcoalition writers on a writer's voting decision in the subsequent year. $\beta_{2}$ measures the influence of out-subcoalition writers on a writer's voting decision in the 
Table 1 BBWAA: Linear Regression Results

\begin{tabular}{ccc}
\hline & $(1)$ & $(2)$ \\
& $v_{i, j, t+1}$ & $v_{i, j, t+1}$ \\
\hline$v_{i, j, t}$ & $0.634^{* * *}$ & $0.634^{* * *}$ \\
$\beta_{1}$ & $(0.0178)$ & $(0.0178)$ \\
& $0.315^{* * *}$ & $0.283^{* * *}$ \\
$\beta_{2}$ & $(0.0278)$ & $(0.0367)$ \\
$\beta_{3}$ & $0.127^{* * *}$ & $0.242^{* * *}$ \\
$\beta_{4}$ & $(0.0265)$ & $(0.0319)$ \\
& & 0.0342 \\
& & $(0.0426)$ \\
Observations & & $-0.135^{* * *}$ \\
R-squared & 19,813 & $(0.0359)$ \\
\hline
\end{tabular}

Robust standard errors in parentheses

*** $p<0.01,{ }^{* *} p<0.05,{ }^{*} p<0.1$

subsequent year. $\delta$ measures the persistence of a writer's voting decision. $\lambda_{t}$ is a set of year fixed effects. Then, we examine whether the pattern of influence changes post-2013 using the following model:

$$
\begin{aligned}
v_{i, j, t+1}= & \delta v_{i, j, t}+\beta_{1} E\left(v_{i, j, t} \mid \text { in }\right)+\beta_{2} E\left(v_{i, j, t} \mid \text { out }\right)+ \\
& \beta_{3} E\left(v_{i, j, t} \mid \text { in }\right) \times \text { Post }-2013+\beta_{4} E\left(v_{i, j, t} \mid \text { out }\right) \times \text { Post } 2013+\lambda_{t}+\epsilon_{i, j, t}
\end{aligned}
$$

$\beta_{3}$ and $\beta_{4}$ measure the change in in- and out-subcoalition influence, respectively, after 2013. Twoway cluster robust standard errors are reported for all regressions, where errors are clustered on the level of the writer and candidate.

The results, which are presented in Table 1, are consistent with a self-reinforcing split among the writers after 2013. Model 1 indicates that writers are more influenced by in-subcoalition writers than by out-subcoalition writers, because $\beta_{1}$ exceeds $\beta_{2}$. However, Model 2 demonstrates that this difference is isolated to the post-2013 period. Prior to 2013, writers are about equally influenced by in- and out-subcoalition writers, consistent with the absence of meaningful subcoalitions in the data. After 2013, however, they become significantly less influenced by out-subcoalition writers. The strength of the influence from in-subcoalition writers does not significantly change. The difference between $\beta_{3}$ and $\beta_{4}$ is significant at the 0.01-level, which indicates that the change in influence from out-subcoalition writers is different from the change in influence from in-subcoalition writers.

sCCA thus reveals a noteworthy change in the social structure of Hall of Fame voting following the 2013 election. Prior to 2013, conflict over candidates was idiosyncratic and writers were influenced equally by those with whom they had similar preferences as those with whom they had 
differing preferences. After 2013, not only did the writers split into two subcoalitions, one sensitive to PED use and one insensitive to it, but also this split appears self-reinforcing, because writers' future voting decisions are no longer as strongly influenced by voters outside of their subcoalition as by voters inside their subcoalition.

These analyses exemplify how sCCA can reveal the evolution of conflict within organizations. By applying sCCA over multiple years, we are able to explore the emergence and persistence of conflict systems. Further, applying sCCA longitudinally enables additional analyses that explore the social dynamics of conflict. In this case, a bifurcation of the BBWAA emerged in 2013 based on divergent views about suspected PED users that persisted through at least 2016. By embedding the results of sCCA in a dynamic regression model, we also show that conflict among the PEDsensitive and PED-insensitive subcoalitions was self-reinforcing, implying that the structure of the conflict among the writers was likely to continue to persist.

\section{Discussion and Conclusion}

In introducing his vision of the business firm as a political coalition, March (1962) expressed hope that by transforming verbal theory on intraorganizational conflict into "computer models of organizational decision making...it is quite likely that we will be able to expose both the theory of the firm as a coalition and the theory of political coalitions in general to new analytic attention" (p. 677). Sixty years hence, management researchers are still waiting for computational methods capable of efficiently identifying conflict systems that are meaningful, representative, and stable. This is the major contribution of sCCA. Given data on individual preferences over desired outcomes, sCCA returns an equilibrium partition of agents into subcoalitions in which the subcoalitions have meaningful preferences and agents are assigned to the subcoalition whose preferences they most agree with. In so doing, sCCA overcomes many of the mathematical and computational challenges faced by prior methods proposed in coalitional game theory. For any set of agents, preferences, and alternatives, sCCA is not only guaranteed to return a solution but also discovers that solution quickly.

As shown in three case studies, sCCA succeeds in identifying latent fault lines among agents in settings where the BTOF's bottom-up conception of organizational conflict is a good fit. In the community ranking of sociology departments, it identifies a small cadre of participants whose views indeed departed from the majority's. In the case of Wikipedia, it predicts the conflict between experienced and newer editors that erupted upon the introduction of the new editing platform. In the study of the BBWAA, sCCA identifies the emergence of conflict in a longitudinal setting by partitioning writers into PED-sensitive and PED-insensitive camps beginning in 2013. The case studies also show how sCCA can be used as an input into subsequent analyses of the structure 
of social conflict by estimating statistical models that predict subcoalition membership and the dynamics of social influence.

sCCA is just one step in a broader effort to build a set of computational tools for revealing the social structure of conflict systems in organizations. We are cautiously optimistic that new developments in computational social choice and political economics will permit more complex political interactions to be analyzed in which agent preferences are endogenous to inter-subcoalition interactions and in which agents' decisions to attach to subcoalitions are driven in part by the desire to secure side payments or achieve individual goals. Perhaps by relying on different social welfare functions or different search algorithms, methods can also be discovered that improve upon the weak stability equilibrium concept in behaviorally-valid and computationally-efficient ways. Finally, new innovations in community detection and machine learning may discover ways to endogenously reveal the number of coalitions in the data, in place of ex post routines like the elbow test, gap statistic, and clustering stability score. That said, these are hard problems with mathematical constraints related to equilibrium existence and computational constraints related to the massive combinatorial space that needs to be searched.

However, we cannot overemphasize the value of a simplified model of coalition-based conflict for testing theories that integrate more complex individual incentives, social institutions, formal roles, and hierarchical relationships. We look to the field of legislative studies in political science as inspiration for how sCCA can be used in this manner. Empirical research on legislative bargaining in political science exploded after Poole and Rosenthal (1985) proposed a computational framework for using roll call votes to assign legislators ideal points on the left-right spectrum. By assuming sincere voting and unidimensional spatial preferences - and thus ignoring strategic voting, party influence, parliamentary rules, and legislative organization - these (DW-)NOMINATE scores reflect a much-simplified model of legislative bargaining. However, precisely for this reason, this framework became the dominant analytical engine for evaluating richer theories of legislative bargaining. We hope that sCCA (or a more worthy successor) can serve this same purpose for the study of coalition-based politics in non-legislative settings.

Along these lines, there exists the potential for sCCA to be used to study coalition structure and formation in other research settings. While we conceive of sCCA as evaluating conflict inside of organizations, it can also straightforwardly apply to modeling conflict within communities of organizations, e.g., social movement industries (McCarthy and Zald 1977) or business groups (Zuckerman and Sgourev 2006). sCCA can also be used, more generally, to identify preference conflict-driven fault lines in any group or population and, as discussed in section 2, may be a valuable complement to computational methods designed to reveal cultural schema. Lastly, we cannot ignore the 
potential for sCCA to also be applied to legislative decision making, particularly in parliamentary settings where the structure of conflict has many dimensions and in which parties enter into coalitions in order to agitate for their platforms.

We conclude by spotlighting the extraordinary opportunity for future work that brings research on intraorganizational conflict together with new methods from computational social choice. Recent research that ties axiomatic results from social choice and game theory in economics together with analyses of efficient algorithms for combinatorial optimization in computer science offers new solutions to the decades-old problem of modeling conflict systems in organizations and firms. We suspect that Richard Cyert, James March, and others at the Carnegie School in the 1950s and 1960s could not foresee how hard it would be to design a computer program to do something as simple as partitioning agents into subcoalitions according to their divergent desires for the future of their firms. The good news for management researchers interested in the Neo-Carnegie approach to the study of politics in organizations is that at least a few of the conceptual and computational hurdles that previously stood in the way of developing new approaches for analyzing observational data on intrafirm conflict are, at long last, being overcome. 


\section{References}

Ahuja RK, Magnanti TL, Orlin JB (1993) Network flows: theory, algorithms, and applications (Englewood Cliffs, N.J: Prentice Hall), ISBN 978-0-13-617549-0.

Arthur D, Vassilvitskii S (2007) k-means++: The advantages of careful seeding. Proceedings of the eighteenth annual ACM-SIAM symposium on Discrete algorithms, 1027-1035 (Society for Industrial and Applied Mathematics), ISBN 0-89871-624-1.

Atkinson N, Ganz SC (2021) Informative Social Choice. mimeo .

Axelrod R, Mitchell W, Thomas RE, Bennett DS, Bruderer E (1995) Coalition Formation in StandardSetting Alliances. Management Science 41(9):1493-1508, ISSN 0025-1909, 1526-5501, URL http:// $\mathrm{dx} . \mathrm{doi} . \mathrm{org} / 10.1287 / \mathrm{mnsc} .41 .9 .1493$

Aziz H, Savani R, Moulin H (2016) Hedonic Games. Brandt F, Conitzer V, Endriss U, Lang J, Procaccia AD, eds., Handbook of Computational Social Choice, 356-376 (Cambridge: Cambridge University Press), ISBN 978-1-107-44698-4, URL http://dx.doi.org/10.1017/CB09781107446984.016.

Bartholdi J, Tovey CA, Trick MA (1989) Voting schemes for which it can be difficult to tell who won the election. Social Choice and Welfare 6(2):157-165, ISSN 1432-217X, URL http://dx.doi.org/10. $1007 / \mathrm{BF} 00303169$

Baumgartner FR, Jones BD (2009) Agendas and Instability in American Politics (Chicago: University of Chicago Press), 2nd edition, ISBN 0226039498 (paper : alk. paper).

Black D (1948) On the Rationale of Group Decision-making. Journal of Political Economy 56(1):23-34, ISSN 0022-3808, URL www.jstor.org/stable/1825026.

Bogomolnaia A, Jackson MO (2002) The Stability of Hedonic Coalition Structures. Games and Economic Behavior 38(2):201-230, ISSN 08998256, URL http://dx.doi.org/10.1006/game.2001.0877.

Boutyline A (2017) Improving the Measurement of Shared Cultural Schemas with Correlational Class Analysis: Theory and Method. Sociological Science 4:353-393, ISSN 23306696, URL http://dx.doi.org/ $10.15195 / v 4.215$

Chalkiadakis G, Elkind E, Wooldridge M (2011) Computational Aspects of Cooperative Game Theory. Synthesis Lectures on Artificial Intelligence and Machine Learning 5(6):1-168, ISSN 1939-4608, 19394616, URL http://dx.doi.org/10.2200/S00355ED1V01Y201107AIM016.

Chappell B (2014) Sportswriter Who Let Readers Fill Out Hall Of Fame Ballot Is Banned. URL https://www.npr.org/sections/thetwo-way/2014/01/09/261229164/ sportswriter-who-let-readers-fill-out-hall-of-fame-ballot-is-banned.

Cohen MD (1984) Conflict and Complexity: Goal Diversity and Organizational Search Effectiveness. The American Political Science Review 78(2):435-451, ISSN 00030554, 15375943, URL http://dx.doi. org/10.2307/1963374. 
Conitzer V, Rognlie M, Xia L (2009) Preference Functions that Score Rankings and Maximum Likelihood Estimation. IJCAI, 109-115, URL http://ijcai.org/Proceedings/09/Papers/029.pdf.

Cox GW, McCubbins MD (1993) Legislative leviathan: party government in the House (Berkeley, California: University of California Press), ISBN 978-0-520-91076-8 978-0-585-07807-6, URL http://search.ebscohost.com/login.aspx?direct=true\&scope=site\&db=nlebk\&db=nlabk\& AN=5782, oCLC: 42856055 .

Cyert RM, March JG (1963) A behavioral theory of the firm. Englewood Cliffs, NJ 2.

Fligstein N (1990) The Transformation of Corporate Control (Cambridge, Mass: Harvard University Press), ISBN 978-0-674-90358-6.

Fligstein N (1993) The transformation of corporate control (Cambridge, Mass.: Harvard Univ. Press), 1. paperback ed edition, ISBN 978-0-674-90359-3 978-0-674-90358-6.

Ford L Jr, Fulkerson D (1962) Flows in Networks (Princeton: Princeton University Press).

Gavetti G, Levinthal D, Ocasio W (2007) Perspective-Neo-Carnegie: The Carnegie School's Past, Present, and Reconstructing for the Future. Organization Science 18(3):523-536.

Goldberg A (2011) Mapping Shared Understandings Using Relational Class Analysis: The Case of the Cultural Omnivore Reexamined. American Journal of Sociology 116(5):1397-1436, ISSN 0002-9602, 1537-5390, URL http://dx.doi.org/10.1086/657976.

Goldberg A, Stein SK (2018) Beyond Social Contagion: Associative Diffusion and the Emergence of Cultural Variation. American Sociological Review 83(5):897-932, ISSN 0003-1224, 1939-8271, URL http://dx. doi.org/10.1177/0003122418797576.

Gupte M, Shankar P, Li J, Muthukrishnan S, Iftode L (2011) Finding hierarchy in directed online social networks. Proceedings of the 20th international conference on World wide web, 557-566 (Hyderabad, India: ACM), ISBN 978-1-4503-0632-4.

Hambrick DC, Mason PA (1984) Upper Echelons: The Organization as a Reflection of Its Top Managers. The Academy of Management Review 9(2):193, ISSN 03637425, URL http://dx.doi.org/10.2307/ 258434 .

Hastie T, Tibshirani R, Friedman JH (2009) The elements of statistical learning: data mining, inference, and prediction. Springer series in statistics (New York, NY: Springer), 2nd ed edition, ISBN 978-0-38784857-0 978-0-387-84858-7.

Healy K (2013) Sociology Department Rankings for 2013. URL https://orgtheory •wordpress .com/2013/ 03/25/sociology-department-rankings-for-2013/.

Honore BE, Kyriazidou E (2000) Panel Data Discrete Choice Models with Lagged Dependent Variables. Econometrica 68(4):839-874, ISSN 0012-9682, 1468-0262, URL http://dx.doi.org/10.1111/ 1468-0262.00139. 
James B (1994) The Politics of Glory: How baseball's Hall of Fame really works (New York : Toronto : New York: Macmillan ; Maxwell Macmillan Canada ; Maxwell Macmillan International), ISBN 978-0-02510774-8.

Keri J (2013) The Fallacy of the Baseball Hall of Fame. URL http://grantland.com/the-triangle/ the-fallacy-of-the-baseball-hall-of-fame/.

Krehbiel K (1997) Pivotal Politics: A Theory of U.S. Lawmaking (Chicago: University of Chicago Press), ISBN 0226452727 (paper : alk. paper).

March JG (1962) The Business Firm as a Political Coalition. Journal of Politics 24(4):662-678.

March JG, Olsen JP (1989) Rediscovering institutions: the organizational basis of politics (New York [etc.]: The Free press), ISBN 978-0-02-920115-2.

McCarthy JD, Zald MN (1977) Resource Mobilization and Social Movements: A Partial Theory. American Journal of Sociology 82(6):1212-1241, ISSN 0002-9602, URL http://www.jstor.org/stable/ 2777934 .

Mintzberg H (1983) Power in and around organizations. The Theory of management policy series (Englewood Cliffs, N.J: Prentice-Hall), ISBN 0-13-686857-6.

Mithani MA, O'Brien JP (2020) So What Exactly Is a "Coalition" Within an Organization? A Review and Organizing Framework. Journal of Management 014920632095043, ISSN 0149-2063, 1557-1211, URL http://dx.doi.org/10.1177/0149206320950433.

Newman MEJ (2006) Modularity and community structure in networks. Proceedings of the National Academy of Sciences 103(23):8577-8582, ISSN 0027-8424, 1091-6490, URL http://dx.doi.org/10.1073/pnas. 0601602103 .

Orlowski A (2013) Revolting peasants force Wikipedia to cut'n'paste Visual Editor into the bin. URL https: //www.theregister.co.uk/2013/09/25/wikipedia_peasants_revolt/.

Pfeffer J, Salancik GR (1974) Organizational Decision Making as a Political Process: The Case of a University Budget. Administrative Science Quarterly 19(2):135, ISSN 00018392, URL http://dx.doi.org/10. $2307 / 2393885$.

Pfeffer J, Salancik GR (2003) The external control of organizations: a resource dependence perspective. Stanford business classics (Stanford, Calif: Stanford Business Books), ISBN 978-0-8047-4789-9.

Poole KT, Rosenthal H (1985) A Spatial Model for Legislative Roll Call Analysis. American Journal of Political Science 29(2):357, ISSN 00925853, URL http://dx.doi.org/10.2307/2111172.

Ray D, Vohra R (2015) Coalition Formation. Handbook of Game Theory with Economic Applications, volume 4, 239-326 (Elsevier), ISBN 978-0-444-53766-9, URL http://dx.doi.org/10.1016/ B978-0-444-53766-9.00005-7.

Roth AE (1988) Introduction to the Shapley value. Roth AE, ed., The Shapley value: essays in honor of Lloyd S. Shapley, 1-30 (Cambridge: Cambridge University Press), ISBN 978-0-521-36177-4. 
Salganik MJ, Levy KEC (2015) Wiki Surveys: Open and Quantifiable Social Data Collection. PLOS ONE 10(5):e0123483, ISSN 1932-6203, URL http://dx.doi.org/10.1371/journal.pone.0123483.

Sampson T (2013) Will Wikipedia's pretty new editing software solve its recruitment crisis? URL https: //www.dailydot.com/business/wikipedia-visual-editor-wysiwyg/.

Silver N (2013) In Cooperstown, a Crowded Waiting Room. URL https://fivethirtyeight.blogs. nytimes.com/2013/01/12/in-cooperstown-a-crowded-waiting-room/

Stevenson WB, Pearce JL, Porter LW (1985) The Concept of "Coalition" in Organization Theory and Research < sup/>. Academy of Management Review 10(2):256-268, ISSN 0363-7425, 1930-3807, URL http://dx.doi.org/10.5465/amr.1985.4278178.

Suh B, Convertino G, Chi EH, Pirolli P (2009) The singularity is not near: slowing growth of Wikipedia. Proceedings of the 5th International Symposium on Wikis and Open Collaboration - WikiSym '09, 1 (Orlando, Florida: ACM Press), ISBN 978-1-60558-730-1, URL http://dx.doi.org/10.1145/ 1641309.1641322 .

Tatti N (2017) Tiers for Peers: a practical algorithm for discovering hierarchy in weighted networks. Data Mining and Knowledge Discovery 31(3):702-738, ISSN 1384-5810, 1573-756X, URL http://dx.doi. org/10.1007/s10618-016-0485-7.

Thompson JD (1967) Organizations in Action: Social Science Bases of Administrative Theory (New Brunswick: Transaction Publishers), ISBN 0-7658-0991-5.

Tibshirani R, Walther G, Hastie T (2001) Estimating the Number of Clusters in a Data Set via the Gap Statistic. Journal of the Royal Statistical Society. Series B (Statistical Methodology) 63(2):411-423, ISSN 1369-7412, URL https://www.jstor.org/stable/2680607.

von Luxburg U (2010) Clustering Stability: An Overview. Foundations and Trends@ in Machine Learning 2(3):235-274, ISSN 1935-8237, URL http://dx.doi.org/10.1561/2200000008.

Von Neumann J, Morgenstern O (1944) Theory of Games and Economic Behavior (Princeton, N.J.: Princeton University Press), ISBN 978-0-691-13061-3, oCLC: ocm78989126.

Wales J (2009) State of the Wiki. URL https://commons.wikimedia.org/wiki/File:State_of_the_ Wiki.pdf.

Williamson DP (2019) Network flow algorithms (Cambridge, United Kingdom ; New York, NY, USA: Cambridge University Press), ISBN 978-1-107-18589-0 978-1-316-63683-1.

Zald MN, Berger MA (1978) Social Movements in Organizations: Coup d'Etat, Insurgency, and Mass Movements. American Journal of Sociology 83(4):823-861, ISSN 00029602, 15375390, URL http: //www.jstor.org/stable/2777718, publisher: University of Chicago Press.

Zuckerman E, Sgourev S (2006) Peer Capitalism: Parallel Relationships in the U.S. Economy. American Journal of Sociology 111(5):1327-1366, ISSN 0002-9602, URL http://dx.doi.org/10.1086/ajs.2006. 111. issue-5. 